\title{
Assessment of groundwater quality for irrigation use: a peninsular case study
}

\author{
Kishan S. Rawat ${ }^{1}$. Sudhir Kumar Singh ${ }^{2} \cdot$ Sandeep Kumar Gautam ${ }^{3}$
}

Received: 2 January 2017 / Accepted: 1 November 2018 / Published online: 26 November 2018

(c) The Author(s) 2018

\begin{abstract}
The grade of irrigation water available to irrigators has a significant impact on crops as well as yields. Therefore, it is a need to better understand irrigation water quality. The present study mainly focuses on the assessment of the suitability of water of forty-four fixed bore wells of Kanchipuram district, Tamil Nadu, India. The groundwater sample datasets of post-monsoon (2005-2013) and pre-monsoon (2006-2013) season were collected for 9 years. Water quality indices, namely sodium adsorption ratio, exchangeable sodium percent (SSP or \% Na), residual sodium carbonate (RSC or RA), Kelly's ratio, permeability index, chloroalkaline indices (CAI1 and CAI2), potential salinity (PS), magnesium hazard, total dissolved solids and total hardness, have been calculated for separate bore wells. The $r_{1}$ and $r_{2}$ indices show that groundwater of the study area is $\mathrm{Na}^{+}-\mathrm{SO}_{4}{ }^{2-}$ and deep meteoric percolation type. Majority of the wells are fall under moderate to unsuitable category of water for irrigation purposes. Further, wells water has also been classified on the base of meteoric genesis index.
\end{abstract}

Keywords Irrigation water quality $\cdot$ Meteoric genesis index $\cdot$ Sodium adsorption ratio $\cdot$ Magnesium adsorption ratio $\cdot$ Geochemistry

\section{Introduction}

Groundwater has become the major source of water use in the agricultural sector in many countries where river and drainage systems are not sufficient. Therefore, poor groundwater quality for irrigation purpose is a matter of worry in recent years. Under or over chemical fertilization is resulting in groundwater pollution (Ayers and Westcot 1985; Rowe and Abdel-Magid 1995; Singh et al. 2013; Gautam et al. 2015, 2016; Nemčić-Jurec et al. 2017). Groundwater quality depends on the nature of recharging water, precipitation,

Sudhir Kumar Singh

sudhirinjnu@gmail.com

Kishan S. Rawat

ksr.kishan@gmail.com

Sandeep Kumar Gautam

kgmsandeep@gmail.com

1 Centre for Remote Sensing and Geo-Informatics, Sathyabama University, Chennai, Tamilnadu 119, India

2 K. Banerjee Centre for Atmospheric and Ocean Studies, IIDS, University of Allahabad, Prayagraj, U.P. 211002, India

3 Centre of Advanced Study in Geology, University of Lucknow, Lucknow 226007, India subsurface and surface water and hydro-geochemical processes in aquifers (Keesari et al. 2016a; Das et al. 2017), land-use/land-cover change (Amin et al. 2014; Srivastava et al. 2013; Gajbhiye et al. 2015; Nemčić-Jurec et al. 2017; Rawat et al. 2017; Rawat and Singh 2018) and mining activities (Gautam et al. 2016; Keesari et al. 2016b; Gautam et al. 2018). Temporal changes in the constitution and origin of the water recharged, and human factor, frequently cause periodic changes in groundwater quality (Vasanthavigar et al. 2010; Milovanovic 2007).

Groundwater quality degrades in twofold, first, due to geochemical reactions in the aquifers and soils and, second, time when it is supplied through improper canals/drainages for irrigation. Therefore, it is necessary to perform a regular assessment of irrigation and drinking water quality (Gupta et al. 2009; Jacintha et al. 2016; Rawat et al. 2018; Gautam et al. 2018). Irrigation demands sufficient water supply of usable quality. The index based on composition and concentration of dissolved elements in water can be useful in determining its applicability for agricultural utilization (Gautam et al. 2013; Singh et al. 2013, 2015). The suitability of groundwater for irrigation depends on the nature of the mineral elements in the water and their impacts on both the soil and plants (Richards 1954; Singh et al. 2009). 
The excess of salts affects plant's growth by redressing the uptake power of plant due to complex changes arouse out of the osmotic processes (Todd 1980).

Generally, water quality parameters (major cations as $\left.\mathrm{Na}^{+}, \mathrm{Ca}^{2+}, \mathrm{Mg}^{2+}, \mathrm{K}^{+}\right)$and anions $\mathrm{Cl}^{-}, \mathrm{SO}_{4}{ }^{2-}, \mathrm{HCO}_{3}{ }^{-}$, $\mathrm{CO}_{3}{ }^{2-}, \mathrm{NO}_{3}{ }^{-}$) and heavy metals are indicators of drinking water use, while water quality indices such as sodium adsorption ratio (SAR), sodium percentage (SSP; \% Na), residual sodium carbonate (RSC), residual alkalinity (RA), Kelly's ratio (KR) [or Kelly's index (KI)], permeability index (PI), chloroalkaline indices (CAI1 and CAI2), potential salinity (PS), magnesium hazard (MH) (or magnesium adsorption ratio; MAR), total dissolved solids (TDS) and total hardness (TH) based on primary water quality parameters are frequently used to determine quality of water for irrigation (Singh et al. 2013, 2015; Gautam et al. 2015).

In the present study, forty-four groundwater samples collected from bore wells were analyzed and assessed for temporal variation and change in water quality index over a period of time. Most of the bore wells are from agricultural areas.

The relation between irrigation and groundwater resources is highly interlinked. This paper describes the groundwater quality status for irrigation purpose using water quality index (SAR, \%Na, RSC, PI, MH, CAI1 and CAI2 CR, TDS, TH, Gibb's 1 and 2) based on primary parameters (such as $\mathrm{K}^{+}, \mathrm{Ca}^{2+}, \mathrm{Cl}^{-}, \mathrm{Na}^{+}, \mathrm{Mg}^{2+}, \mathrm{NO}_{3}{ }^{-}, \mathrm{SO}_{4}{ }^{2-}$ and $\mathrm{HCO}_{3}{ }^{-}$). Further, the classification was performed based on Soltan method and estimation of groundwater source based on meteoric genesis index. Therefore, the aim of the study was to determine suitability of goundwater for irrigation.

\section{Study area}

Kanchipuram district of Tamil Nadu (India) state lies between $11^{\circ} 00^{\prime}$ and $12^{\circ} 00^{\prime}$ north latitudes and $77^{\circ} 28^{\prime}-78^{\circ} 50^{\prime}$ east longitudes (North East coast of Tamil Nadu) and on the banks of the Vegavathi River, a tributary of the Palar River (Fig. 1a). The study area has an elevation of $83.2 \mathrm{~m}$ above mean sea level. The land around study area is flat and slopes toward the south and east. It is bound by Bay of Bengal in the east.

Agriculture is the main occupation of the people with $47 \%$ of the population engaged in it. Paddy is the major crop cultivated in this district. Industrial developments occupy around 65 ha (160 acres), where most of the handloom spinning, silk weaving, dyeing and rice production units are located. 89.06 ha (220.1 acres) are used for transport and communications infrastructure, including bus stands, roads, streets and railways lines. Groundnuts, sugarcane, cereals and millets and pulses are the other major crops (Table 1). The soil in the region is mostly clay, with some loam, clay and sand (Table 1). The total forest area in the district is $23,586 \mathrm{ha}$, and it spreads in the interior region and around the district. In this forest area, there are 366.675 ha of reserved land. Totally, 76.50 metric tonnes lands are cultivated in fuelwood and 8.039 tonnes in cashew.

The pre-monsoon rainfall is almost uniform throughout the district. The coastal regions get more rains rather than the interior regions. This district is mainly depending on the seasonal rains, and the distress conditions prevail in the event of the failure of rains. Northeast and southwest monsoons are the major donors with $54 \%$ and $36 \%$ contribution each to the total annual rainfall. During normal monsoon, the district receives a rainfall of $1213.3 \mathrm{~mm}$ (Table 1).

\section{Materials and methods}

The data related to groundwater quality have been acquired from Chennai Water Metro Board/Central Ground Water Board (CGWB) during pre- and post-monsoon seasons. Totally, 44 samples were collected during May 2005 (premonsoon) and sampling activity was repeated during January 2006 (post-monsoon) of period 2005-2013. Well coordinates have been collected using a handheld GPS device (eTrex Legend ${ }^{\circledR} \mathrm{HCx}$, having 10-m accuracy).

\section{Development of rainfall datasets using satellite datasets}

The precipitation data of the entire study area have been derived from daily precipitation data provided by the NOAA climate prediction center and were downloaded from the site ftp://ftpprd.ncep.noaa.gov/pub/cpc/fews/S.Asia/. The need of satellite-estimated precipitation arises because of the nondependable and poorly spatially distributed ground rainfall data (Rawat and Tripathi 2016; Rawat et al. 2012a; b, 2016). Daily precipitation satellite data have been converted into monthly precipitation data (Fig. 1b) by simply sum of per day rainfall of particular month (Rawat and Tripathi 2016; Rawat et al. 2012c, d, 2016).

\section{Irrigation indices}

\section{Sodicity}

Sodic soils are characterized by a disproportionately high concentration of sodium $\left(\mathrm{Na}^{+}\right)$in their cation exchange complex. Sodicity is the effect of irrigation water and can alter the chemical and physical properties of the soil due to an accumulation of $\mathrm{Na}^{+}$. Excess of $\mathrm{Na}^{+}$can affect plants in three ways: (1) by degrading soil structure after each rainfall and irrigation due to crust formation which reduces water movement (permeability) and aeration in the soil; (2) toxic effects when absorbed by leaves/roots; and (3) $\mathrm{K}^{+}$and $\mathrm{Ca}^{2+}$ 
(a)

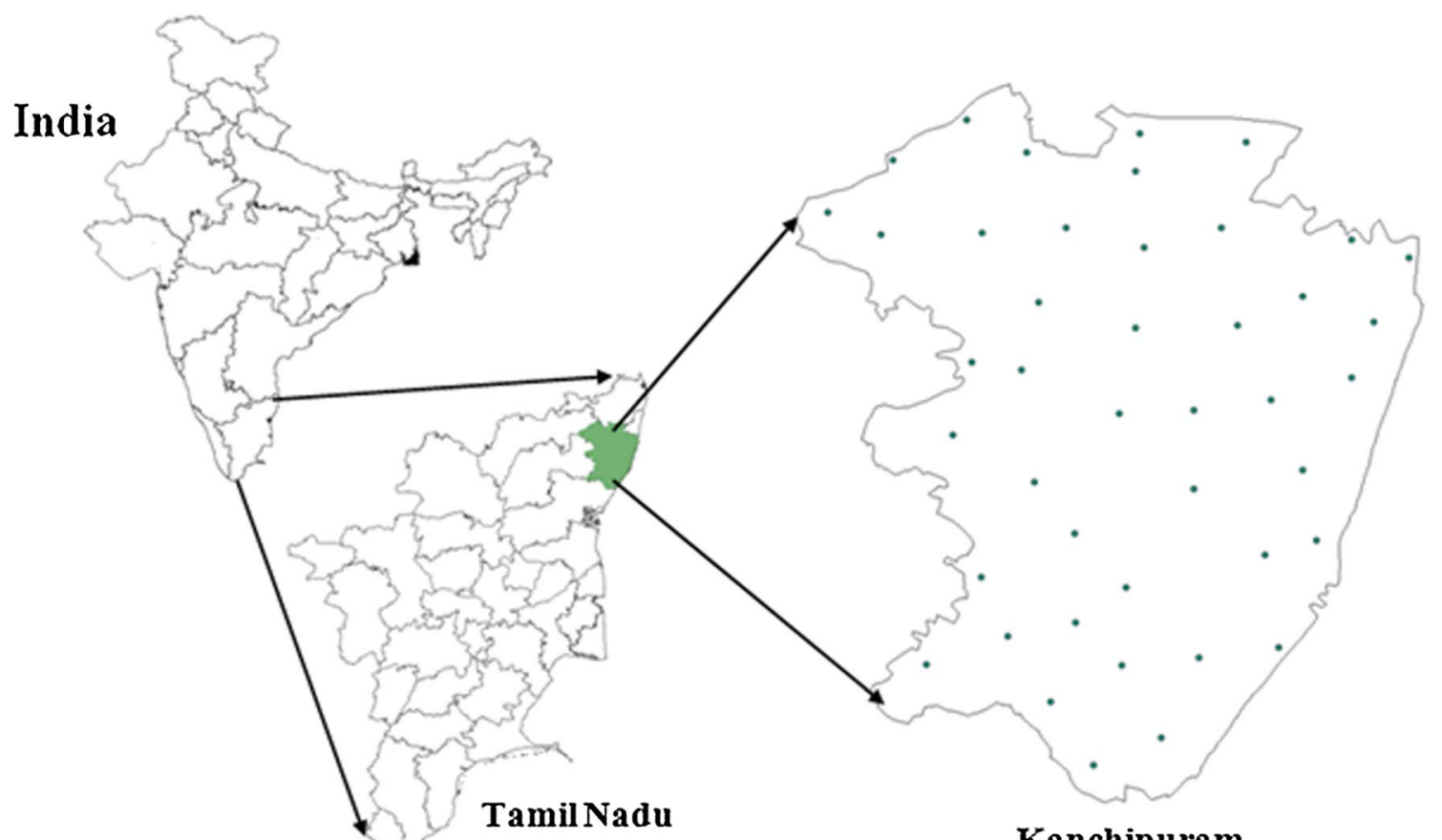

Kanchipuram

(b)

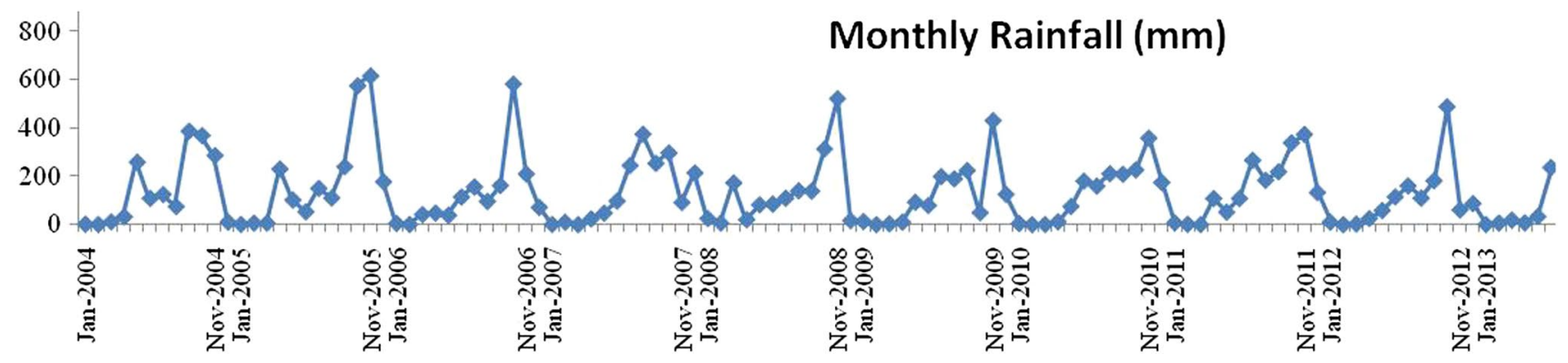

Fig. 1 a Study area map with 44 sampling locations. b Graphical representation of rainfall variability in the study area during 2005-2013

deficiencies may arise if the soil or irrigation water has a high concentration of $\mathrm{Na}^{+}$. Therefore, evaluation of the sodicity hazard of irrigation water is important.

\section{Sodium adsorption ratio (SAR)}

The SAR is a relative ratio of $\mathrm{Na}^{+}$ions to $\mathrm{Ca}^{2+}$ and $\mathrm{Mg}^{2+}$ ions present in the water sample. The SAR is used to estimate the potential of $\mathrm{Na}^{+}$to accumulate in the soil primarily (water movement) at the expense of $\mathrm{Ca}^{2+}, \mathrm{Mg}^{2+}$ and $\mathrm{K}^{+}$as a result of regular use of sodic water. It is formulated as Eq. (1):

$\mathrm{SAR}=\frac{\mathrm{Na}^{+}}{\sqrt{\frac{\left(\mathrm{Ca}^{2+}+\mathrm{Mg}^{2+}\right)}{2}}}$

where $\mathrm{Na}^{+}, \mathrm{Ca}^{2+}$ and $\mathrm{Mg}^{2+}$ are in meq/l.
On the basis of SAR range, irrigation water can be classified into four classes as SAR $<10$ (ideal or excellent), 10-18 (good), 18-26 (doubtful) and $>26$ (unsuitable).

SAR also influences percolation time of water in the soil. Therefore, the low value of SAR of irrigation water is desirable.

\section{Residual sodium carbonate (RSC)/residual alkalinity (RA)}

RSC represent as the amount of sodium carbonate $\left(\mathrm{NaCO}_{3}\right)$ and sodium bicarbonate $\left(\mathrm{NaHCO}_{3}\right)$ present in the irrigation water if the concentration of carbonate $\left(\mathrm{CO}_{3}{ }^{2-}\right)$ and bicarbonate $\left(\mathrm{HCO}_{3}{ }^{-}\right)$ions exceeds the concentrations of $\mathrm{Ca}^{2+}$ and $\mathrm{Mg}^{2+}$ ions (Raghunath 1987), precipitation of $\mathrm{Ca}^{2+}$ and $\mathrm{Mg}^{2+}$. If the carbonates are less than alkaline earths $\left(\mathrm{Ca}^{2+}+\mathrm{Mg}^{2+}\right)$, it outlined the residual $\mathrm{NaCO}_{3}$ which is absent. Generally, RSC is expressed as milliequivalents 
Table 1 General information about the study area (Source: www.kanchi.tn.nic.in)

\begin{tabular}{ll}
\hline Total area & $4393.37 \mathrm{~km}^{2}$ \\
Net sown area & $1364.89 \mathrm{~km}^{2}$ \\
Net irrigation area & $1236.28 \mathrm{~km}^{2}$ \\
Forest area & $426.57 \mathrm{~km}^{2}$ \\
Poromboke area & $1553.47 \mathrm{~km}^{2}$ \\
Town area & $82.57 \mathrm{~km}^{2}$ \\
Number of panchayat villages & 648 \\
Summer temperature & $21.1-36.6^{\circ} \mathrm{C}$ \\
Winter temperature & $19.8-28.7{ }^{\circ} \mathrm{C}$ \\
Rainfall & $1133.0 \mathrm{~mm}$ actual, 1213.3 mm normal \\
Major crops & Rice (145,966 ha), sugarcane (7586 ha), \\
& groundnut (28,766 ha), gingelly (912 ha), \\
& pulses (2966 ha), cotton (53 ha), millets and \\
Soil types & cereals (1217 ha) \\
& Read loam (Kanchipuram, Uthiramerur blocks), \\
& lateritic soil (plateaus in the district), black \\
& soil (spread in all blocks), sandy coastal \\
& alluviam (Thiruporur and St. Thomas Mount) \\
and red sandy soil (Kancheepuram and urban
\end{tabular}

per liter (meq/l) of $\mathrm{NaCO}_{3}$. An excess of $\mathrm{CO}_{3}{ }^{2-}$ and $\mathrm{HCO}_{3}{ }^{-}$causes precipitation of soil $\mathrm{Ca}^{2+}$ and $\mathrm{Mg}^{2+}$ impairing the soil structure as well as potentially activating soil sodium. On the basis of RSC range, sodium hazard has been classified into three classes as follows: RSC $<1.25$ (low), $1.25-2.5$ (medium) and $>2.5$ (high). RSC is expressed as Eq. (2):

$\mathrm{RSC}=\left(\mathrm{HCO}_{3}^{-}+\mathrm{CO}_{3}^{2-}\right)-\left(\mathrm{Ca}^{2+}+\mathrm{Mg}^{2+}\right)$

A high range of $\mathrm{RSC}$ in irrigation water means an increase in the adsorption of sodium on the soil. Water having $\mathrm{RSC}>5$ has not been recommended for irrigation because of damaging effects on plant growth. Generally any source of water in which RSC is higher than 2.5 is not considered suitable for agriculture purpose, and water $<1.25$ is recommended as safe for irrigation purpose. A negative value of RSC reveals that concentration of $\mathrm{Ca}^{2+}$ and $\mathrm{Mg}^{2+}$ is in excess. A positive RSC denotes that $\mathrm{Na}^{+}$existences in the soil are possible. RSC calculation is also important in context to calculate the required amount of gypsum or sulfuric acid per acre-foot in irrigation water to neutralize residual carbonates effect.

\section{Percent sodium (\%Na) or sodium hazard}

The $\% \mathrm{Na}$ is also used in classifying water for irrigation purpose. $\mathrm{Na}^{+}$is important parameter and helps in categorization of any source of water for irrigation uses. $\mathrm{Na}^{+}$makes chemical bounding with soil to reduce water movement capacity of the soil (Ayers and Westcot 1985). Percent $\mathrm{Na}^{+}$concentration is a factor to assess its suitability for irrigation purposes
(Wilcox 1948). $\mathrm{Na}^{+}$reacts with $\mathrm{CO}_{3}{ }^{2-}$ and forms alkaline soils, while $\mathrm{Na}^{+}$reacts with chloride and forms saline soils. Sodium-affected soil (alkaline/saline) retards crop growth (Todd 1980). If concentration of $\mathrm{Na}^{+}$in irrigation water is high, then the ions tend toward the clay particles, by removing $\mathrm{Ca}^{2+}$ and $\mathrm{Mg}^{2+}$ ions through a base-exchange reaction. This exchange process in soil reduces water movement capacity. In this condition, air and water cannot move freely or restricted during wet conditions, and such soils have become hard when dry (Collins and Jenkins 1996; Saleh et al. 1999). The \% Na values are calculated as Eq. (3):

$\% \mathrm{Na}=\frac{\mathrm{Na}^{+}}{\mathrm{Ca}^{2+}+\mathrm{Mg}^{2+}+\mathrm{Na}^{+}+\mathrm{K}^{+}} \times 100$

(all the ion concentrations are expressed in meq/l).

The classification of water is based on $\% \mathrm{Na}$ as excellent $(<20 \%)$, good $(20-40 \%)$, permissible (40-60\%), doubtful (60-80\%) and unsuitable (>80\%) (Khodapanah et al. 2009).

\section{Kelly's ratio (KR) or Kelly's index (KI)}

Kelly (1940) and Paliwal (1967) introduced another factor to assess quality and classification of water for irrigation purpose based on the concentration of $\mathrm{Na}^{+}$against $\mathrm{Ca}^{2+}$ and $\mathrm{Mg}^{2+}$. It can be calculated using Eq. (4)

$\mathrm{KR}=\frac{\mathrm{Na}^{+}}{\mathrm{Ca}^{2+}+\mathrm{Mg}^{2+}}$

(all the ion concentrations are expressed in meq/l).

$\mathrm{KR} / \mathrm{KI}>1$ indicates an excess level of $\mathrm{Na}^{+}$in waters. Therefore, water with a $\mathrm{KI} \leq 1$ has been recommended for 
irrigation, while water with $\mathrm{KI} \geq 1$ is not recommended for irrigation due to alkali hazards (Ramesh and Elango 2012; Karanth 1987).

\section{Permeability index (PI)}

The permeability index (PI) is an indicator to study the suitability water for irrigation purpose. Water movement capability in soil (permeability) is influenced by the long-term use of irrigation water (with a high concentration of salt) as it is affected by $\mathrm{Na}^{+}, \mathrm{Ca}^{2+}, \mathrm{Mg}^{2+}$ and $\mathrm{HCO}_{3}{ }^{-}$ions of the soil. PI formula has been developed by Doneen (1964), to assess water movement capability in the soil as the suitability of any kind of source of water for irrigation, and it is formulated as Eq. (5):

$\mathrm{PI}=\frac{\mathrm{Na}^{+}+\sqrt{\mathrm{HCO}_{3}^{-}}}{\mathrm{Ca}^{2+}+\mathrm{Mg}^{2+}+\mathrm{Na}^{+}} \times 100$

(all the ion concentrations are expressed in meq/l).

According to Doneen (1964), PI can be categorized in three classes: class I ( $>75 \%$, suitable), class II $(25-75 \%$, good) and class III ( $<25 \%$, unsuitable). Water under class I and class II is recommended for irrigation.

\section{Magnesium hazard (MH) or magnesium adsorption ratio (MAR)}

Usually, alkaline earths $\left(\mathrm{Ca}^{2+}\right.$ and $\left.\mathrm{Mg}^{2+}\right)$ are in an equilibrium state in groundwater. Both $\mathrm{Ca}^{2+}$ and $\mathrm{Mg}^{2+}$ ions are linked with soil friability and aggregation, but both are also essential nutrients for the crop. The high value of $\mathrm{Ca}^{2+}$ and $\mathrm{Mg}^{2+}$ in water can increase soil $\mathrm{pH}$ (therefore soil converting it to saline nature of the soil; Joshi et al. 2009), resulting in decrease in the availability of phosphorous (Al-Shammiri et al. 2005). Excess concentration of magnesium in groundwater affects the soil quality by converting it into alkaline and decreases the crop yield (Gowd 2005; Singh et al. 2013; Gautam et al. 2015). According to agriculturists, excess amount of $\mathrm{Mg}^{2+}$ ions in waters damage the soil quality which causes low crop production (Ramesh and Elango 2012; Narsimha et al. 2013). Szabolcs and Darab (1964) projected MH values for irrigation water, and it is calculated using Eq. (6):

$\mathrm{MH}=\frac{\mathrm{Mg}^{2+}}{\mathrm{Ca}^{2+}+\mathrm{Mg}^{2+}} \times 100$

(all the ion concentrations are expressed in meq/l).

$\mathrm{MH}>50$ is not recommended for irrigation purposes (Khodapanah et al. 2009).

\section{Potential salinity (PS)}

PS is another water quality parameter-based index (Doneen 1964) for categorization of water for agriculture use. $\mathrm{PS}<3 \mathrm{meq} / \mathrm{l}$ is an indication of the suitability of water for irrigation. The temporal distribution of PS of the study area is produced for pre- and post-monsoon seasons using following Eq. (7):

$\mathrm{PS}=\mathrm{Cl}^{-}+0.5 \times \mathrm{SO}_{4}^{2-}$

\section{Chloroalkaline indices (CAI1 and CAI2)}

Information about coming changes in chemical composition of the groundwater during underground travel is also vital (Sastri 1994). The chemical reaction in which ion exchange between the groundwater and the aquifer occurs during the movement and rest condition of water. It can be analyzed through the chloroalkaline indices. The CAI1 and CAI2 are evaluated (Schoeller 1977) and expressed by Eqs. (8 and 9):

$\mathrm{CAI} 1=\frac{\mathrm{Cl}^{-}-\left(\mathrm{Na}^{+}+\mathrm{K}^{+}\right)}{\mathrm{Cl}^{-}}$

$\mathrm{CAI} 2=\frac{\mathrm{Cl}^{-}-\left(\mathrm{Na}^{+}+\mathrm{K}^{+}\right)}{\left(\mathrm{SO}_{4}^{2-}+\mathrm{CO}_{3}^{2-}+\mathrm{HCO}_{3}^{-}+\mathrm{NO}_{3}^{-}\right)}$

The CAI1 and CAI2 indices may be negative or positive depending on the exchange process of $\mathrm{Na}^{+}$and $\mathrm{K}^{+}$from the rock with $\mathrm{Mg}^{2+}$ and $\mathrm{Ca}^{2+}$ present in water and vice versa. If a direct exchange process (DEP) happens between $\mathrm{Na}^{+}$and $\mathrm{K}^{+}$ in water with $\mathrm{Mg}^{2+}$ and $\mathrm{Ca}^{2+}$ in rocks, then $\mathrm{CAI}$ ratio will be positive. If a reverse exchange process occurs $\left(\mathrm{Na}^{+}\right.$and $\mathrm{K}^{+}$in water with $\mathrm{Mg}^{2+}$ and $\mathrm{Ca}^{2+}$ in rocks), then CAI ratio will be negative.

\section{Corrosivity ratio (CR)}

The corrosivity ratio is giving the information about water supply. Any source of water with $\mathrm{CR}<1$ is recommended to the transport of any source of water in any kind of pipes, whereas CR $>1$ shows corrosive nature of water, means not to be transported through metal pipes (Balasubramanian 1986; Shankar et al. 2011; Aravindan 2004). The CR can be estimated using an Eq. (10):

$\mathrm{CR}=\frac{\left(\frac{\mathrm{Cl}^{-}}{35}\right)+2\left(\frac{\mathrm{SO}_{4}^{2-}}{96}\right)}{\left(\frac{\mathrm{CO}_{3}^{2-}+\mathrm{HCO}_{3}^{-}}{100}\right)}$

(all ions are in ppm). 
The rate of corrosion depends upon some physical parameters like pressure, temperature and rate of flow of water. In addition to the higher value of $\mathrm{Cl}^{-}$and $\mathrm{SO}_{4}{ }^{2-}$ also increases the corrosion rate.

\section{Total dissolved solids (TDS)}

In natural water total dissolved solids contains of minerals, nutrients that have dissolved in water, and also includes major ions, i.e. $\mathrm{Ca}^{2+}, \mathrm{Mg}^{2+}, \mathrm{Na}^{+}, \mathrm{K}^{+}, \mathrm{CO}_{3}{ }^{2-}, \mathrm{HCO}_{3}{ }^{-}, \mathrm{Cl}^{-}$, $\mathrm{SO}_{4}{ }^{2-}$ and $\mathrm{PO}_{4}{ }^{3-}$. Weathering or dissolution of soil and rocks generates ions in water (Singh et al. 2013). After evaporation of water, accumulation of salt at the root zone makes obstacle and plants are not capable of sucking water from soil resulting in moisture stress (Modi 2000). For irrigation, the TDS has been classified as TDS $<450 \mathrm{mg} / \mathrm{l}$ and is preferred for irrigation and TDS $>450-2000 \mathrm{mg} / \mathrm{l}$ is slight to moderate and TDS $>2000 \mathrm{mg} / \mathrm{l}$ is unsuitable for agricultural purpose (FAO 2006).

\section{Total hardness (TH)}

Water hardness is a result of existence of divalent metallic cations $\left(\mathrm{Ca}^{2+}\right.$ and $\left.\mathrm{Mg}^{2+}\right)$, and it can be calculated as the sum of $\mathrm{Ca}^{2+}$ and $\mathrm{Mg}^{2+}$ concentration as meq/l equivalent to $\mathrm{CaCO}_{3}$ (Todd 1980) and expressed by (Eq. 11):

$\mathrm{TH}=2.5 \times \mathrm{Ca}^{2+}+4.1 \times \mathrm{Mg}^{2+}$

The high value of TH is due to encrustation on water supply systems (pipes) for distribution. Moderate value of TH is useful for plumbing system from corrosion. Total hardness classified as $100 \mathrm{mg} / \mathrm{l}$ provides good control over corrosion and is the usually acceptable limit. TH is usually classified (EPA $1986)$ as soft $(0-60 \mathrm{mg} / \mathrm{l})$, moderately hard $(60-120 \mathrm{mg} / \mathrm{l})$, hard $(120-180 \mathrm{mg} / \mathrm{l})$ and very hard $(>180 \mathrm{mg} / \mathrm{l})$.

\section{Classification of groundwater}

Soltan (1998) suggested the classification of different sources of water facies based on the concentration (meq/l) of particular ion. Soltan (1999) suggested a new classification method based on base-exchange indices, and it can be calculated using Eq. (12)

$r_{1}=\frac{\mathrm{Na}^{+}-\mathrm{Cl}^{-}}{\mathrm{SO}_{4}^{2-}}$

The groundwater of the study area can be categorized as $\mathrm{Na}^{+}-\mathrm{HCO}_{3}{ }^{-}$type if $r_{1}>1$ and $\mathrm{Na}^{+}-\mathrm{SO}_{4}{ }^{2-}$ type if $r_{1}<1$.

The groundwater has also been categorized based on meteoric genesis index, which was calculated (Soltan 1999) using Eq. (13).
$r_{2}=\frac{\left(\mathrm{K}^{+}+\mathrm{Na}^{+}\right)-\mathrm{Cl}^{-}}{\mathrm{SO}_{4}^{2-}}$

If the value of $r_{2}<1$ it means the groundwater is of deep meteoric percolation (DMP) type, and if the value of $r_{2}>1$ it means the groundwater is of shallow meteoric percolation (SMP) type.

\section{Result and discussion}

\section{Irrigation indices}

Figure $2 \mathrm{a}, \mathrm{b}$ shows the fluctuation of SAR value during preand post-monsoon season and Tables 2 and 3 show 9-year statistics. Tables 2 and 3 revealed that SAR value ranges from 0.25 to 69.09 and 0.24 to 78.37 in pre- and post-monsoon, respectively. The average value of SAR for 9 years was reported as 18.74 and 17.49 which are the nearest average value (18.93 and 19.17 and 17.30 and 17.40) of 2010 and 2011 (pre-monsoon) and 2006 and 2008 (post-monsoon). According to individual year statistics, 2007 and 2012 have lowest average value of SAR (15.92 and 15.91) during post-monsoon. It is due to good amount of rainfall (Fig. 1b, 7- and 8-month rainfall out of 12 during 2007 and 2012, respectively). The same year's range of SAR was also lowest (1.05-46.14 and 1.94-67.93). Similarly, Table 3 shows 32 wells ( 15 excellent, 31.82 percent +17 good, 38.64 percent), and 32 wells (19 excellent, 43.18 percent +13 good, 39.55 percent) fall under excellent and good category during the years 2007 and 2012 (post-monsoon). The rainfall affects SAR value of the year 2006 (pre-monsoon). The 2007 postmonsoon saw a good amount of rainfall as 70.45 percent (19 excellent + 12 good) wells have reported normal SAR limit, whereas in 2011 pre-monsoon, majority of water went as runoff (less amount of infiltration into groundwater); hence, in 2012 pre-monsoon, only 21 wells ( 17 excellent +4 good) fell under safe limit of SAR. From Fig. 2a, during pre-monsoon well nos. 4, 12, 21, 27, 34 and 40 were cross the higher (>26) limit of SAR, respectively, 9, 7, 6, 7, 3 and 7 time (year) out of 9 years, and it highlights that these wells are not appropriate for pre-monsoon irrigation. From Fig. 2b, these same wells have higher SAR value as $>26$ (limit) with $7,6,4,3,0$ and 8 times out of 8 years during post-monsoon. Therefore, these wells $(4,12,21,27$ and 40$)$ were unsuitable during the study period (2005-2013).

The RSC value ranges from -0.10 to 6.87 with an average value of 1.76 over 2005-2013 during pre-monsoon (Table 2), while during post-monsoon RSC value ranges from -4.73 to 5.04 (for 8 years) with 1.488 -year average (Table 3). From Table 4 in years 2011 and 2012, wells ( $47.73 \%$ and $65.91 \%$, respectively) fall under the good limit 


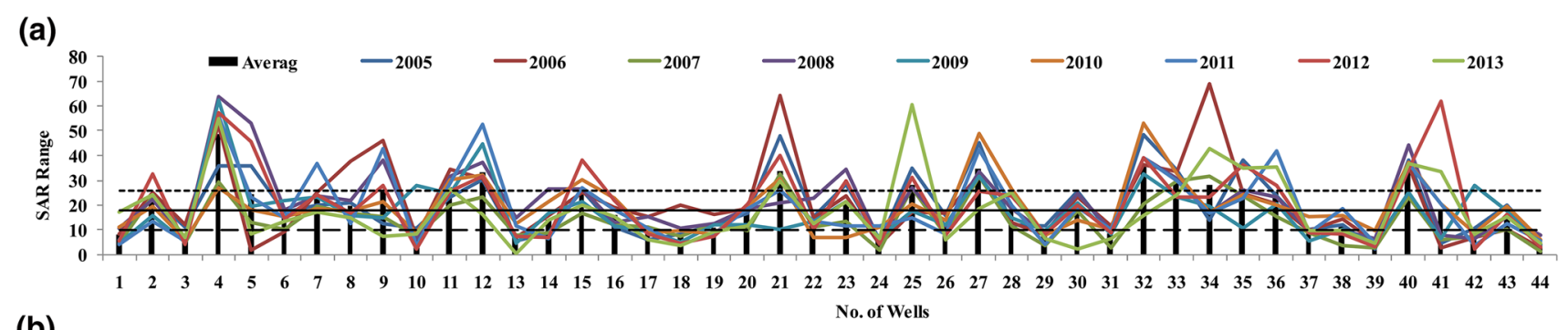

(b)

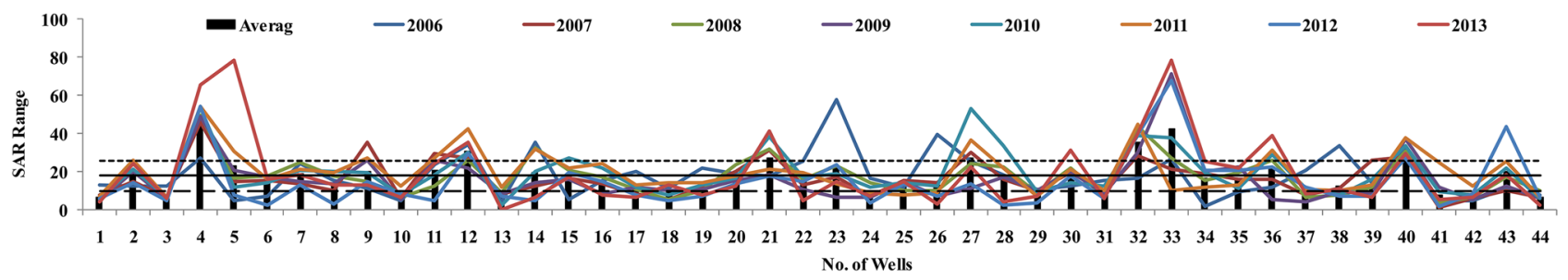

Fig. 2 a Nine-year (2005-2013) temporal representation of SAR value during pre-monsoon. b Nine-year (2006-2013) temporal representation of SAR value during post-monsoon

$(<1.25)$ due to good amount of monthly rainfall during 2010 and 2011 (above $200 \mathrm{~mm}$ ), which provide higher rate of infiltration rather than runoff. Similarly, during post-monsoonof year 2013, highest no. of wells $(59.09 \%$, Table 5) falls under the good limit $(<1.25)$ of RSC. It shows during 2013 factor $\left(\mathrm{HCO}_{3}{ }^{-}, \mathrm{CO}_{3}{ }^{2-}, \mathrm{Ca}^{2+}\right.$ and $\left.\mathrm{Mg}^{2+}\right)$ which govern $\mathrm{RSC}$ were less in groundwater due to slow leaching from rock to groundwater. Effect of rainfall over RSC can be easily understood from Fig. 3a, b, well nos. 2, 4, 5, 12, 21, 25, 32,35 and 41 most of the time of year come under beyond of the unsuitable limit during pre-monsoon, but after rainfall or due to rainfall effect these well's RSC range reduces. In Fig. 3b, some wells $(6,24,32,36$ and 44$)$ having negative RSC values due to excess concentration of $\mathrm{Ca}^{2+}$ and $\mathrm{Mg}^{2+}$. A high range of RSC in groundwater indicates an increase in the adsorption of sodium in soil (Eaton 1950) during irrigation from such type of bore well. It is observed that well nos. 2, 4, 5, 12, 21, 25, 32, 35 and 41 fall under unsuitable category for irrigation purpose because of RSC value $>2.5 \mathrm{meq} / \mathrm{l}$ is harmful for plants growth.

From Tables 2 and 3, 9-year average of \% Na found 55.04 (with $\min =3.27$ and $\max =87.59$ ) and 54.38 for pre- and post-monsoon, respectively, and both values are under the permissible limit for irrigation. Individual yearwise study shows the years 2006 (during pre-monsoon) and 2011 (during post-monsoon) average value of $\% \mathrm{Na}[60.05$ (premonsoon) and 57.30 (post-monsoon)] was high during the study period. Statistical analysis (Tables 2,3 ) of $\% \mathrm{Na}$ does not show much variation in $\% \mathrm{Na}$ values yearwise, and also Tables 2 and 3 revealed that all the year wise statistical values (AV, Me, Mo, Mi, Ma and SD) showing most no. of wells falls under acceptable limit of \%Na. From Table 4 , it is clear that majority of percent of wells come under excellent, good and permissible category, but the doubtful category also reports the good percent of total no. of wells during the pre-monsoon years 2006, 2010 and 2011, respectively, $45.45,45.45$ and $59.09 \%$. It may be due to low rainfall and leads to slow dilution process. Table 5 shows the doubtful category (except years 2006 and 2010) during post-monsoon and explain rainfall effect over $\% \mathrm{Na}$. Figure $4 \mathrm{a}, \mathrm{b}$ also explains the effect of rainfall over groundwater quality in the context of $\% \mathrm{Na}$. Only well no. 33 showing a negative effect of rainfall, it may be due to more leaching of $\mathrm{Na}^{+}$ from the rock into the water. Overall, Fig. $4 \mathrm{~b}$ represents dilution in \% Na with respect to Fig. 4a during the study period 2005-2013. Based on Fig. 4a, b, majority of wells come in the good and permissible category of irrigation water.

Kelly's ratio (KR)/Kelly's index (KI) is an indicator to asses irrigation water suitability and it is free from the effect of $\mathrm{K}^{+}$parameter, which purely depends on $\mathrm{Ca}^{2+}, \mathrm{Mg}^{2+}$ and $\mathrm{Na}^{+}$. Its classification bin (only two classes) is also easier than $\% \mathrm{Na}$ classification bin (four classes). From Tables 2 and 3, average value of KR/KI during the study period was 1.39 and 1.34 for pre- and post-monsoon, respectively, with a range of 0.03-6.66 and 0.03-12.31. Statistical analysis of KR has revealed that majority of wells falls in unsuitable category during pre-monsoon. Except for year 2007 KR varies from $0.03-3.63$ with average KR was found 0.92 (Table 2) during pre-monsoon. After analysis of post-monsoon KR value, it was found that there was very less effect of rainfall over KR because after rainfall average value of KR (Table 3) comes under the suitable range due to dilution process. Table 4 denotes that during the pre-monsoon study period more than 52 percent wells in the study area come under the suitable category $(\mathrm{KR}<1)$ of irrigation except the years 2005 and 2007. Table 5 does not take into

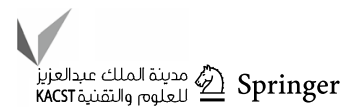


Table 2 Statistics of each index during pre-monsoon

\begin{tabular}{|c|c|c|c|c|c|c|c|c|c|c|}
\hline & 2005 & 2006 & 2007 & 2008 & 2009 & 2010 & 2011 & 2012 & 2013 & N YA \\
\hline \multicolumn{11}{|l|}{$S A R$} \\
\hline AV & 19.25 & 21.15 & 13.88 & 21.13 & 17.17 & 18.93 & 19.17 & 20.66 & 17.34 & 18.74 \\
\hline $\mathrm{Me}$ & 15.51 & 17.10 & 11.48 & 18.30 & 15.50 & 17.79 & 14.88 & 20.41 & 14.29 & 15.80 \\
\hline Mo & 10.42 & 7.00 & 7.30 & 6.51 & 28.25 & 8.41 & 3.77 & 2.48 & 8.07 & 12.73 \\
\hline $\mathrm{Mi}$ & 2.09 & 1.83 & 1.13 & 5.43 & 3.81 & 5.79 & 3.77 & 2.48 & 0.25 & 0.25 \\
\hline $\mathrm{Ma}$ & 48.49 & 69.09 & 32.42 & 63.78 & 62.60 & 53.23 & 55.81 & 61.95 & 60.63 & 69.09 \\
\hline SD & 12.70 & 15.61 & 9.01 & 13.22 & 11.39 & 10.86 & 13.81 & 14.95 & 13.67 & 13.01 \\
\hline \multicolumn{11}{|c|}{$R S C$} \\
\hline AV & 1.82 & 1.74 & 1.64 & 2.00 & 1.64 & 1.88 & 1.75 & 1.61 & 1.78 & 1.76 \\
\hline $\mathrm{Me}$ & 1.61 & 1.65 & 1.49 & 1.86 & 1.63 & 1.83 & 1.43 & 0.98 & 1.52 & 1.59 \\
\hline Мо & 1.27 & 0.31 & 0.27 & 1.23 & 1.00 & 0.61 & 2.19 & 0.46 & 0.40 & 1.27 \\
\hline Mi & 0.10 & -0.10 & 0.27 & 0.15 & 0.02 & 0.01 & 0.04 & 0.01 & 0.32 & -0.10 \\
\hline Ma & 6.42 & 5.21 & 3.85 & 4.90 & 3.67 & 5.07 & 5.58 & 6.87 & 5.62 & 6.87 \\
\hline SD & 1.29 & 1.08 & 0.96 & 1.23 & 0.91 & 1.10 & 1.22 & 1.53 & 1.26 & 1.18 \\
\hline \multicolumn{11}{|c|}{$\% N a$} \\
\hline AV & 57.42 & 60.05 & 46.98 & 58.50 & 53.11 & 55.75 & 57.66 & 55.64 & 50.22 & 55.04 \\
\hline $\mathrm{Me}$ & 56.09 & 62.30 & 47.60 & 58.56 & 55.87 & 58.65 & 59.86 & 55.86 & 53.84 & 56.02 \\
\hline Мо & 55.17 & 87.59 & 45.24 & 44.78 & 61.92 & 48.81 & 33.33 & 53.83 & 39.04 & 44.78 \\
\hline Mi & 20.35 & 22.73 & 10.47 & 30.41 & 26.26 & 35.17 & 27.93 & 29.26 & 3.27 & 3.27 \\
\hline $\mathrm{Ma}$ & 85.94 & 87.59 & 81.90 & 86.02 & 85.86 & 84.94 & 86.46 & 79.10 & 86.95 & 87.59 \\
\hline SD & 15.17 & 16.53 & 16.44 & 13.87 & 14.56 & 12.53 & 14.61 & 12.51 & 18.49 & 15.45 \\
\hline \multicolumn{11}{|c|}{$K R / K I$} \\
\hline AV & 1.55 & 1.55 & 0.92 & 1.57 & 1.26 & 1.33 & 1.36 & 1.66 & 1.29 & 1.39 \\
\hline $\mathrm{Me}$ & 1.18 & 1.29 & 0.76 & 1.15 & 1.06 & 1.17 & 1.10 & 1.18 & 0.89 & 1.06 \\
\hline Mo & 1.18 & 1.17 & 0.76 & 0.76 & 1.56 & 0.91 & 0.28 & 0.28 & 0.64 & 1.17 \\
\hline $\mathrm{Mi}$ & 0.16 & 0.09 & 0.09 & 0.38 & 0.27 & 0.49 & 0.28 & 0.27 & 0.03 & 0.03 \\
\hline $\mathrm{Ma}$ & 5.78 & 5.43 & 3.63 & 5.35 & 6.00 & 5.17 & 4.26 & 5.56 & 6.66 & 6.66 \\
\hline SD & 1.28 & 1.12 & 0.71 & 1.13 & 1.15 & 0.84 & 1.00 & 1.39 & 1.21 & 1.12 \\
\hline \multicolumn{11}{|l|}{$P I$} \\
\hline AV & 62.41 & 62.75 & 51.18 & 63.47 & 57.54 & 60.90 & 59.33 & 61.27 & 57.16 & 59.56 \\
\hline $\mathrm{Me}$ & 60.39 & 65.35 & 51.36 & 60.39 & 58.50 & 61.39 & 61.95 & 58.68 & 55.37 & 59.47 \\
\hline Mo & 63.75 & 71.79 & 53.76 & 56.75 & 65.34 & 60.00 & 33.49 & 40.11 & 47.32 & 63.75 \\
\hline Mi & 25.08 & 18.03 & 18.61 & 36.51 & 29.13 & 39.39 & 33.49 & 30.75 & 12.10 & 12.10 \\
\hline Мa & 99.42 & 88.55 & 87.43 & 89.75 & 90.09 & 90.42 & 86.56 & 91.24 & 94.69 & 99.42 \\
\hline SD & 14.89 & 15.79 & 15.68 & 13.55 & 13.76 & 11.58 & 14.99 & 17.85 & 16.70 & 15.36 \\
\hline \multicolumn{11}{|l|}{$M H$} \\
\hline AV & 51.99 & 49.90 & 44.98 & 42.90 & 37.33 & 32.83 & 35.76 & 44.10 & 38.32 & 42.01 \\
\hline $\mathrm{Me}$ & 46.46 & 50.92 & 47.58 & 42.75 & 35.02 & 32.02 & 33.33 & 39.84 & 37.11 & 39.46 \\
\hline Mo & 33.33 & 22.22 & 39.13 & 50.00 & 33.33 & 20.93 & 33.33 & 37.79 & 37.79 & 33.33 \\
\hline Mi & 21.05 & 14.02 & 4.65 & 16.67 & 11.96 & 13.16 & 5.51 & 18.55 & 17.28 & 4.65 \\
\hline $\mathrm{Ma}$ & 82.98 & 92.98 & 72.88 & 75.56 & 69.36 & 59.49 & 79.66 & 74.81 & 68.35 & 92.98 \\
\hline SD & 17.60 & 18.96 & 14.04 & 13.70 & 12.70 & 10.60 & 15.63 & 16.10 & 11.82 & 15.87 \\
\hline \multicolumn{11}{|l|}{$P S$} \\
\hline AV & 2.36 & 2.66 & 2.43 & 2.70 & 2.63 & 2.59 & 2.78 & 2.36 & 2.13 & 2.52 \\
\hline $\mathrm{Me}$ & 1.94 & 2.09 & 1.95 & 1.86 & 1.91 & 1.52 & 2.04 & 1.91 & 1.35 & 1.85 \\
\hline Mo & 0.94 & 0.39 & 0.80 & 0.56 & 4.89 & 2.18 & 3.74 & 0.38 & 1.04 & 1.23 \\
\hline $\mathrm{Mi}$ & 0.25 & 0.37 & 0.40 & 0.47 & 0.36 & 0.40 & 0.41 & 0.38 & 0.15 & 0.15 \\
\hline Ma & 7.78 & 8.99 & 7.30 & 11.31 & 9.54 & 10.83 & 12.32 & 9.99 & 6.58 & 12.32 \\
\hline SD & 1.94 & 2.14 & 1.78 & 2.35 & 2.13 & 2.43 & 2.51 & 1.84 & 1.68 & 2.10 \\
\hline \multicolumn{11}{|c|}{ CIA1 } \\
\hline AV & -0.040 & -0.107 & 0.233 & -0.033 & 0.129 & -0.122 & 0.120 & 0.122 & 0.092 & 0.04 \\
\hline
\end{tabular}


Table 2 (continued)

\begin{tabular}{|c|c|c|c|c|c|c|c|c|c|c|}
\hline & 2005 & 2006 & 2007 & 2008 & 2009 & 2010 & 2011 & 2012 & 2013 & N YA \\
\hline $\mathrm{Me}$ & 0.102 & 0.206 & 0.391 & 0.166 & 0.279 & 0.079 & 0.176 & 0.226 & 0.295 & 0.22 \\
\hline Мо & 0.415 & -3.080 & 0.367 & 0.143 & 0.344 & 0.047 & 0.267 & -0.107 & 0.345 & -0.11 \\
\hline Mi & -2.438 & -3.080 & -2.256 & -2.043 & -1.258 & -2.120 & -0.930 & -1.800 & -3.339 & -3.34 \\
\hline Ma & 0.814 & 0.826 & 0.908 & 0.775 & 0.764 & 0.784 & 0.802 & 0.795 & 0.951 & 0.95 \\
\hline SD & 0.688 & 0.859 & 0.588 & 0.641 & 0.513 & 0.722 & 0.377 & 0.520 & 0.743 & 0.65 \\
\hline \multicolumn{11}{|c|}{ CIA2 } \\
\hline AV & 0.18 & 0.12 & 0.24 & 0.17 & 0.25 & 0.13 & 0.19 & 0.23 & 0.16 & 0.19 \\
\hline $\mathrm{Me}$ & 0.04 & 0.09 & 0.16 & 0.08 & 0.14 & 0.03 & 0.05 & 0.16 & 0.10 & 0.09 \\
\hline Mo & 0.31 & 0.00 & 0.00 & 0.00 & 0.00 & 0.00 & 0.06 & -0.03 & 0.15 & 0.31 \\
\hline Mi & -0.25 & -1.16 & -0.22 & -0.26 & -0.23 & -0.30 & -0.33 & -0.28 & -0.27 & -1.16 \\
\hline Ma & 1.45 & 1.87 & 1.33 & 1.16 & 1.74 & 1.74 & 1.60 & 1.98 & 1.05 & 1.98 \\
\hline SD & 0.37 & 0.48 & 0.33 & 0.35 & 0.45 & 0.42 & 0.37 & 0.42 & 0.29 & 0.39 \\
\hline \multicolumn{11}{|l|}{$C R$} \\
\hline AV & 2.16 & 2.25 & 1.67 & 1.72 & 1.78 & 1.89 & 1.67 & 1.97 & 1.08 & 1.80 \\
\hline $\mathrm{Me}$ & 1.15 & 1.28 & 1.16 & 0.99 & 1.05 & 1.00 & 1.05 & 1.52 & 0.97 & 1.06 \\
\hline Mo & 1.75 & 0.00 & 0.00 & 0.00 & 0.00 & 0.00 & 0.76 & 0.71 & 1.37 & 1.75 \\
\hline Mi & 0.18 & 0.19 & 0.15 & 0.23 & 0.24 & 0.14 & 0.31 & 0.24 & 0.13 & 0.13 \\
\hline $\mathrm{Ma}$ & 23.80 & 19.42 & 13.25 & 13.90 & 12.74 & 19.72 & 8.42 & 14.55 & 3.52 & 23.80 \\
\hline SD & 3.77 & 3.36 & 2.26 & 2.31 & 2.20 & 3.23 & 1.71 & 2.40 & 0.69 & 2.59 \\
\hline \multicolumn{11}{|c|}{$T D S$} \\
\hline AV & 719.91 & 762.27 & 729.77 & 798.89 & 764.43 & 794.98 & 798.43 & 705.86 & 674.92 & 749.94 \\
\hline $\mathrm{Me}$ & 622 & 664 & 587 & 710 & 636 & 618 & 678 & 646.5 & 605 & 648.50 \\
\hline Mo & 253 & 222 & 269 & 206 & 1277 & 240 & 378 & 682 & 387 & 262.00 \\
\hline Mi & 233 & 222 & 235 & 206 & 237 & 238 & 221 & 188 & 113 & 113.00 \\
\hline Ma & 3314 & 2734 & 2955 & 2727 & 2999 & 2608 & 2481 & 2412 & 1405 & 3314.00 \\
\hline SD & 516.81 & 463.87 & 463.43 & 491.76 & 493.54 & 499.44 & 511.18 & 431.89 & 349.15 & 468.70 \\
\hline \multicolumn{11}{|l|}{$T H$} \\
\hline AV & 328.09 & 329.60 & 400.67 & 352.33 & 376.13 & 360.33 & 342.38 & 307.56 & 338.62 & 385.21 \\
\hline $\mathrm{Me}$ & 277.40 & 272.70 & 338.20 & 279.25 & 306.65 & 289.90 & 253.35 & 239.41 & 272.07 & 282.35 \\
\hline Mo & 118.30 & 51.40 & 143.80 & 113.30 & 540.30 & 121.90 & 255.20 & 109.89 & 224.76 & 190.70 \\
\hline Mi & 64.20 & 51.40 & 133.30 & 111.50 & 86.00 & 121.90 & 103.30 & 109.89 & 94.87 & 51.40 \\
\hline Ma & 1495.60 & 1277.80 & 1337.60 & 1167.00 & 1340.80 & 1116.60 & 1419.60 & 1178.30 & 1147.04 & 1495.60 \\
\hline SD & 238.18 & 220.09 & 238.06 & 239.84 & 251.92 & 240.26 & 232.99 & 203.95 & 207.48 & 340.52 \\
\hline \multicolumn{11}{|l|}{$r_{1}$} \\
\hline AV & -0.89 & -0.78 & -1.79 & -1.17 & -1.48 & -0.52 & -1.54 & -0.86 & -1.07 & -1.12 \\
\hline $\mathrm{Me}$ & -0.54 & -0.71 & -1.29 & -0.59 & -0.88 & -0.38 & -1.11 & -0.94 & -0.99 & -0.82 \\
\hline Мo & -1.50 & -0.15 & -0.63 & -0.17 & -0.88 & -0.10 & -0.61 & -0.85 & -0.52 & -0.80 \\
\hline Mi & -10.42 & -6.78 & -12.11 & -13.27 & -10.73 & -9.10 & -12.09 & -8.78 & -14.06 & -14.06 \\
\hline Ma & 7.20 & 10.50 & 8.30 & 2.37 & 2.61 & 3.00 & 7.25 & 9.22 & 14.25 & 14.25 \\
\hline SD & 2.38 & 2.32 & 3.04 & 2.93 & 2.53 & 1.77 & 2.79 & 2.57 & 4.63 & 2.87 \\
\hline \multicolumn{11}{|l|}{$r_{2}$} \\
\hline AV & 0.15 & -0.12 & -1.34 & -0.95 & -1.14 & -0.28 & -0.77 & -0.40 & -0.45 & -0.59 \\
\hline $\mathrm{Me}$ & -0.20 & -0.35 & -0.99 & -0.28 & -0.53 & -0.20 & -0.30 & -0.58 & -0.82 & -0.50 \\
\hline Mo & -1.42 & 3.78 & -0.55 & -0.12 & -0.82 & -0.05 & -0.28 & 0.15 & -0.52 & -0.55 \\
\hline Mi & -10.17 & -6.26 & -10.56 & -17.14 & -10.27 & -9.00 & -17.91 & -8.70 & -13.03 & -17.91 \\
\hline $\mathrm{Ma}$ & 16.50 & 10.50 & 8.80 & 3.26 & 2.83 & 4.50 & 14.00 & 12.00 & 18.88 & 18.88 \\
\hline SD & 3.71 & 2.45 & 2.98 & 3.53 & 2.63 & 1.89 & 3.87 & 3.09 & 5.46 & 3.43 \\
\hline
\end{tabular}

$A v$ average, $M e$ median, $M o$ mode, $M i$ minimum, $M a$ maximum, $S D$ standard deviation, $N Y A$ nine-year average 
Table 3 Statistics of each index during post-monsoon

\begin{tabular}{|c|c|c|c|c|c|c|c|c|c|}
\hline 2005 & 2006 & 2007 & 2008 & 2009 & 2010 & 2011 & 2012 & 2013 & N YA \\
\hline \multicolumn{10}{|l|}{$S A R$} \\
\hline AV & 17.30 & 15.92 & 17.40 & 15.74 & 19.24 & 19.56 & 15.91 & 18.87 & 17.49 \\
\hline $\mathrm{Me}$ & 15.15 & 14.43 & 16.25 & 12.03 & 16.47 & 16.46 & 13.47 & 13.21 & 14.53 \\
\hline Mo & 4.59 & 5.75 & 6.86 & 5.21 & 8.20 & 12.02 & 8.52 & 6.61 & 21.23 \\
\hline Mi & 1.63 & 1.05 & 3.64 & 4.17 & 2.72 & 6.39 & 1.94 & 0.24 & 0.24 \\
\hline $\mathrm{Ma}$ & 57.57 & 46.14 & 49.49 & 71.17 & 53.70 & 54.05 & 67.93 & 78.37 & 78.37 \\
\hline SD & 11.21 & 9.40 & 10.13 & 12.43 & 11.86 & 11.29 & 13.69 & 18.59 & 12.57 \\
\hline \multicolumn{10}{|l|}{$R S C$} \\
\hline AV & 1.18 & 1.99 & 1.88 & 1.68 & 1.79 & 1.08 & 1.46 & 0.86 & 1.48 \\
\hline $\mathrm{Me}$ & 1.38 & 1.81 & 1.93 & 1.56 & 1.89 & 1.23 & 1.43 & .77 & 1.51 \\
\hline Mo & 1.46 & 2.90 & 1.60 & 0.40 & 1.08 & 1.05 & 0.51 & 0.34 & 1.71 \\
\hline Mi & -4.73 & -1.43 & -2.42 & -2.85 & -1.54 & -3.36 & -3.31 & -3.47 & -4.73 \\
\hline Ma & 3.79 & 5.04 & 4.56 & 4.89 & 3.92 & 3.74 & 4.28 & 3.81 & 5.04 \\
\hline SD & 1.56 & 1.35 & 1.44 & 1.33 & 1.22 & 1.26 & 1.53 & 1.21 & 1.41 \\
\hline \multicolumn{10}{|l|}{$\% N a$} \\
\hline $\mathrm{AV}$ & 55.79 & 51.60 & 54.37 & 52.20 & 56.97 & 57.30 & 53.35 & 53.44 & 54.38 \\
\hline $\mathrm{Me}$ & 57.65 & 50.84 & 53.42 & 52.40 & 57.35 & 56.96 & 53.82 & 54.62 & 54.25 \\
\hline Mo & 38.71 & 42.86 & 42.86 & 40.00 & 61.90 & 59.55 & 48.79 & 38.50 & 42.86 \\
\hline Mi & 15.28 & 30.43 & 33.54 & 29.48 & 25.52 & 35.00 & 25.36 & 10.60 & 10.60 \\
\hline Ma & 85.60 & 79.22 & 83.71 & 88.10 & 83.56 & 83.45 & 89.89 & 92.49 & 92.49 \\
\hline SD & 15.40 & 13.00 & 12.61 & 14.11 & 13.06 & 12.51 & 15.29 & 18.84 & 14.48 \\
\hline \multicolumn{10}{|l|}{$K R / K I$} \\
\hline AV & 1.34 & 1.11 & 1.24 & 1.21 & 1.39 & 1.44 & 1.24 & 1.76 & 1.34 \\
\hline $\mathrm{Me}$ & 1.11 & 0.94 & 1.01 & 0.88 & 1.18 & 1.20 & 0.89 & 0.79 & 0.98 \\
\hline Mo & 0.53 & 0.72 & 0.67 & 0.59 & 0.82 & 1.42 & 0.91 & 0.62 & 1.00 \\
\hline Mi & 0.15 & 0.08 & 0.29 & 0.35 & 0.16 & 0.53 & 0.12 & 0.03 & 0.03 \\
\hline $\mathrm{Ma}$ & 4.83 & 3.56 & 4.38 & 7.34 & 4.49 & 4.56 & 8.58 & 12.31 & 12.31 \\
\hline SD & 1.01 & 0.78 & 0.88 & 1.19 & 0.93 & 0.93 & 1.43 & 2.49 & 1.31 \\
\hline \multicolumn{10}{|l|}{$P I$} \\
\hline AV & 59.72 & 57.29 & 59.31 & 57.08 & 62.08 & 61.63 & 55.63 & 57.25 & 58.75 \\
\hline $\mathrm{Me}$ & 60.64 & 56.09 & 57.88 & 54.12 & 62.73 & 61.23 & 57.22 & 51.88 & 57.96 \\
\hline Mo & 47.27 & 56.02 & 49.21 & 51.43 & 58.33 & 68.03 & 59.08 & 48.72 & 47.27 \\
\hline Mi & 30.26 & 25.48 & 36.50 & 30.56 & 20.85 & 36.64 & 16.95 & 19.69 & 16.95 \\
\hline $\mathrm{Ma}$ & 89.38 & 87.11 & 89.42 & 92.00 & 91.82 & 89.01 & 96.08 & 99.19 & 99.19 \\
\hline SD & 14.24 & 13.32 & 12.58 & 13.81 & 13.17 & 12.46 & 16.13 & 19.15 & 14.53 \\
\hline \multicolumn{10}{|l|}{$M H$} \\
\hline AV & 66.37 & 34.99 & 34.66 & 48.28 & 39.70 & 40.97 & 39.11 & 42.30 & 43.30 \\
\hline $\mathrm{Me}$ & 64.31 & 36.14 & 32.63 & 46.78 & 41.46 & 37.99 & 37.79 & 39.61 & 41.03 \\
\hline Mo & 53.85 & 31.25 & 42.03 & 33.33 & 16.00 & 33.33 & 28.83 & 33.29 & 33.33 \\
\hline Mi & 38.64 & 0.85 & 11.69 & 22.87 & 1.64 & 15.29 & 13.78 & 4.82 & 0.85 \\
\hline Мa & 86.44 & 68.75 & 74.63 & 72.17 & 80.00 & 71.76 & 70.85 & 77.74 & 86.44 \\
\hline SD & 13.14 & 12.98 & 12.71 & 14.19 & 17.97 & 14.33 & 13.61 & 17.98 & 17.49 \\
\hline \multicolumn{10}{|l|}{$P S$} \\
\hline AV & 2.54 & 2.51 & 2.52 & 2.20 & 2.62 & 2.91 & 2.30 & 2.08 & 2.46 \\
\hline $\mathrm{Me}$ & 2.27 & 1.77 & 1.54 & 1.63 & 1.63 & 2.04 & 1.50 & 1.32 & 1.67 \\
\hline Mo & 4.03 & 0.72 & 0.51 & 0.50 & 0.49 & 0.99 & 0.70 & 0.67 & 0.66 \\
\hline Mi & 0.21 & 0.21 & 0.35 & 0.38 & 0.34 & 0.74 & 0.32 & 0.00 & 0.00 \\
\hline $\mathrm{Ma}$ & 7.98 & 11.80 & 13.26 & 9.33 & 12.68 & 12.29 & 8.65 & 7.26 & 13.26 \\
\hline SD & 1.94 & 2.56 & 2.69 & 1.75 & 2.68 & 2.41 & 2.17 & 1.87 & 2.28 \\
\hline \multicolumn{10}{|l|}{ CIAI } \\
\hline $\mathrm{AV}$ & 0.13 & -0.03 & -0.04 & 0.16 & -0.11 & 0.31 & -0.01 & 0.23 & 0.08 \\
\hline
\end{tabular}


Table 3 (continued)

\begin{tabular}{|c|c|c|c|c|c|c|c|c|c|}
\hline 2005 & 2006 & 2007 & 2008 & 2009 & 2010 & 2011 & 2012 & 2013 & N YA \\
\hline $\mathrm{Me}$ & 0.25 & 0.22 & 0.15 & 0.20 & -0.01 & 0.31 & 0.21 & 0.27 & 0.21 \\
\hline Мо & 0.00 & 0.14 & -1.00 & 0.19 & -0.41 & 0.38 & 0.21 & 0.45 & 0.00 \\
\hline $\mathrm{Mi}$ & -1.44 & -1.73 & -1.68 & -1.54 & -1.82 & -0.48 & -2.09 & -1.26 & -2.09 \\
\hline Ma & 0.85 & 0.78 & 0.79 & 0.83 & 0.82 & 0.81 & 0.76 & 0.86 & 0.86 \\
\hline SD & 0.54 & 0.70 & 0.59 & 0.46 & 0.63 & 0.25 & 0.64 & 0.44 & 0.56 \\
\hline \multicolumn{10}{|l|}{ CIA2 } \\
\hline AV & 0.39 & 0.17 & 0.20 & 0.26 & 0.18 & 0.46 & 0.19 & 0.34 & 0.27 \\
\hline $\mathrm{Me}$ & 0.12 & 0.07 & 0.05 & 0.09 & 0.00 & 0.19 & 0.08 & 0.20 & 0.09 \\
\hline Mo & 0.00 & 0.00 & 0.05 & 0.00 & -0.07 & 0.00 & 0.08 & 0.29 & 0.00 \\
\hline Mi & -0.28 & -0.21 & -0.28 & -0.22 & -0.26 & -0.18 & -0.47 & -0.75 & -0.75 \\
\hline $\mathrm{Ma}$ & 4.36 & 1.88 & 3.07 & 2.73 & 3.25 & 6.34 & 2.54 & 3.21 & 6.34 \\
\hline SD & 0.87 & 0.39 & 0.59 & 0.58 & 0.60 & 1.01 & 0.54 & 0.63 & 0.68 \\
\hline \multicolumn{10}{|l|}{$C R$} \\
\hline AV & 2.38 & 1.16 & 1.34 & 1.48 & 1.42 & 2.42 & 1.83 & 2.34 & 1.80 \\
\hline $\mathrm{Me}$ & 1.27 & 0.91 & 0.69 & 0.99 & 0.82 & 1.49 & 0.95 & 1.17 & 1.07 \\
\hline Mo & 0.00 & 0.00 & 1.24 & 0.00 & 0.50 & 0.00 & 1.15 & 1.09 & 1.24 \\
\hline $\mathrm{Mi}$ & 0.20 & 0.09 & 0.14 & 0.21 & 0.15 & 0.43 & 0.20 & 0.00 & 0.00 \\
\hline Ma & 14.82 & 6.25 & 9.50 & 10.82 & 8.01 & 16.74 & 14.78 & 17.97 & 17.97 \\
\hline SD & 3.06 & 1.21 & 1.79 & 1.85 & 1.66 & 3.03 & 2.99 & 3.23 & 2.49 \\
\hline \multicolumn{10}{|l|}{$T D S$} \\
\hline AV & 738.43 & 761.91 & 766.02 & 670.43 & 781.98 & 782.84 & 714.77 & 616.77 & 729.14 \\
\hline $\mathrm{Me}$ & 664.5 & 593 & 603.5 & 553 & 627 & 609 & 537 & 499 & 596.00 \\
\hline Мo & 215 & 593 & 279 & 330 & 258 & 258 & 243 & 664 & 321.00 \\
\hline Mi & 170 & 175 & 195 & 204 & 258 & 258 & 243 & 98 & 98.00 \\
\hline $\mathrm{Ma}$ & 2436 & 2614 & 2427 & 1800 & 3087 & 2562 & 2373 & 2520 & 3087.00 \\
\hline SD & 480.37 & 523.41 & 502.77 & 377.54 & 563.96 & 523.82 & 470.45 & 424.95 & 484.92 \\
\hline \multicolumn{10}{|l|}{$T H$} \\
\hline AV & 371.79 & 394.39 & 375.78 & 347.94 & 362.58 & 356.49 & 346.27 & 292.95 & 356.02 \\
\hline $\mathrm{Me}$ & 289.85 & 295.85 & 274.40 & 282.30 & 265.35 & 255.05 & 272.03 & 241.96 & 269.28 \\
\hline Mo & 130.20 & 96.00 & 155.60 & 118.30 & 137.80 & 109.20 & 199.70 & 254.57 & 254.57 \\
\hline Mi & 114.70 & 96.00 & 106.00 & 118.30 & 132.20 & 109.20 & 99.80 & 69.82 & 69.82 \\
\hline Мa & 1646.40 & 1517.00 & 1514.60 & 1298.50 & 1250.20 & 1356.40 & 1358.00 & 1397.71 & 1646.40 \\
\hline SD & 289.80 & 311.99 & 291.19 & 231.76 & 271.30 & 271.20 & 260.32 & 223.61 & 269.12 \\
\hline \multicolumn{10}{|l|}{$r_{1}$} \\
\hline AV & -1.98 & -0.92 & -1.44 & -1.65 & -0.85 & -1.96 & -0.81 & -2.07 & -1.46 \\
\hline $\mathrm{Me}$ & -1.14 & -0.66 & -0.42 & -0.73 & -0.30 & -1.21 & -0.66 & -1.58 & -0.75 \\
\hline Mo & -1.36 & -0.15 & -0.28 & -0.25 & 0.27 & -1.21 & -0.39 & -4.83 & -0.25 \\
\hline Mi & -14.00 & -8.98 & -14.40 & -14.29 & -14.88 & -10.89 & -8.35 & -14.40 & -14.88 \\
\hline Ma & 2.67 & 4.47 & 2.43 & 2.05 & 2.53 & 0.84 & 3.77 & 8.80 & 8.80 \\
\hline SD & 3.36 & 2.40 & 3.83 & 2.91 & 2.74 & 2.12 & 1.70 & 3.73 & 2.95 \\
\hline \multicolumn{10}{|l|}{$r_{2}$} \\
\hline AV & -1.23 & -0.47 & -1.34 & -1.42 & -0.66 & -1.91 & -0.45 & -1.84 & -1.17 \\
\hline $\mathrm{Me}$ & -0.67 & -0.46 & -0.18 & -0.33 & 0.01 & -0.99 & -0.39 & -1.21 & -0.46 \\
\hline Mo & 0.00 & -0.12 & -0.18 & -0.17 & 0.39 & -1.14 & -0.33 & -4.82 & 0.00 \\
\hline Mi & -8.00 & -8.98 & -19.84 & -17.86 & -19.75 & -20.74 & -9.91 & -16.00 & -20.74 \\
\hline Мa & 3.50 & 9.90 & 3.07 & 2.75 & 2.68 & 1.18 & 4.10 & 8.82 & 9.90 \\
\hline SD & 2.80 & 2.88 & 4.88 & 3.36 & 3.442308 & 3.329189 & 1.99 & 3.84 & 3.42 \\
\hline
\end{tabular}

Av average, $M e$ median, $M o$ mode, $M i$ minimum, $M a$ maximum, $S D$ standard deviation, $N Y A$ nine year average 
Table 4 Classification of fixed bore wells water during pre-monsoon within the study area for irrigation based on \%Na, SAR, MH, KR RSC, TDS (Wilcox 1948; Kelly 1940; Todd 1980; USSL 1954)

\begin{tabular}{|c|c|c|c|c|c|c|c|c|c|c|c|}
\hline \multirow[t]{2}{*}{ Index } & \multirow[t]{2}{*}{ Range } & \multirow[t]{2}{*}{ Class } & \multicolumn{9}{|c|}{ No. of samples (with \%) under different classes per year } \\
\hline & & & 2005 & 2006 & 2007 & 2008 & 2009 & 2010 & 2011 & 2012 & 2013 \\
\hline \multirow[t]{4}{*}{ SAR } & $<10$ & Exc. & $14,31.82$ & $13,29.55$ & $19,43.18$ & 11,25 & $13,29.55$ & 11,25 & 11,25 & $17,38.64$ & $17,38.64$ \\
\hline & $10-18$ & Go. & 11,25 & 11,25 & $12,27.27$ & $10,22.73$ & $13,29.55$ & 11,25 & 11,25 & $04,9.10$ & $10,22.73$ \\
\hline & $18-26$ & Dou. & $08,18.18$ & $9,20.45$ & $07,15.91$ & $10,22.73$ & $12,27.27$ & $13,29.45$ & 11,25 & $9,20.45$ & $07,15.91$ \\
\hline & $>26$ & UnSu. & 11,25 & 11,25 & $6,13.64$ & $13,29.55$ & $06,13.64$ & $09,20.45$ & 11,25 & $14,31.82$ & $10,22.73$ \\
\hline \multirow[t]{3}{*}{ RSC/RA } & $<1.25$ & Go. & $16,36.36$ & $14,31.82$ & $13,29.55$ & $16,36.36$ & $18,40.91$ & $12,27.27$ & $21,47.73$ & $29,65.91$ & $17,38.62$ \\
\hline & $1.25-2.5$ & Dou. & $17,38.64$ & $9,20.45$ & $23,52.27$ & $16,36.36$ & $16,36.36$ & 22,50 & $14,31.82$ & $7,15.91$ & $19,43.18$ \\
\hline & $>2.5$ & UnSu. & 11,25 & $21,47.73$ & $08,18.18$ & $12,27.27$ & $10,22.73$ & $10,22.73$ & $09,20.45$ & $08,18.18$ & $08,18.18$ \\
\hline \multirow[t]{5}{*}{$\% \mathrm{Na}$} & $>20$ & Exce. & $01,2.27$ & $01,2.27$ & $04,9.09$ & 00,00 & 00,00 & 00,00 & 00,00 & 00,00 & 00,00 \\
\hline & $20-40$ & Go. & $06,13.64$ & $04,9.09$ & $07,15.91$ & $02,4.54$ & $09,20.45$ & $04,9.09$ & $04,9.09$ & $03,6.82$ & $13,29.5$ \\
\hline & $40-60$ & Perm. & $21,47.73$ & $15,34.09$ & 22,50 & 22,50 & $23,52.27$ & $19,43.18$ & $16,36.36$ & $26,59.09$ & $17,38.64$ \\
\hline & $60-80$ & Dou. & $13,29.55$ & $20,45.45$ & $10,22.73$ & $17,38.64$ & $10,22.73$ & $20,45.45$ & $20,45.45$ & $15,34.09$ & $10,22.73$ \\
\hline & $>80$ & UnSu. & $03,6.82$ & $04,9.09$ & $01,2.27$ & $03,6.82$ & $02,4.55$ & $01,2.27$ & $04,9.09$ & 00,00 & $02,4.55$ \\
\hline \multirow[t]{2}{*}{$\mathrm{KR} / \mathrm{Kl}$} & $<1$ & Su. & $19,43.18$ & $31,70.45$ & $13,29.55$ & $25,56.82$ & $24,54.55$ & $24,54.55$ & $23,52.27$ & $23,52.27$ & $24,54.55$ \\
\hline & $>1$ & UnSu. & $25,56.82$ & $13,29.55$ & $31,70.45$ & $19,43.18$ & $20,45.45$ & $20,45.45$ & $21,47.73$ & $21,47.73$ & $20,45.45$ \\
\hline \multirow[t]{3}{*}{ PI } & $>75 \%$ & Su. & $09,20.45$ & $08,18.18$ & $02,4.55$ & $08,18.18$ & $03,6.82$ & $05,11.36$ & $06,13.64$ & 11,25 & $06,13.64$ \\
\hline & $25-75 \%$ & Go. & $34,77.27$ & $35,79.55$ & $38,86.36$ & $36,81.82$ & $41,93.18$ & $39,88.64$ & $38,86.36$ & 33,75 & $37,84.09$ \\
\hline & $<25 \%$ & UnSu. & $01,2.27$ & $01,2.27$ & $04,9.09$ & 00,00 & 00,00 & 00,00 & 0,00 & 00,00 & $1,2.27$ \\
\hline \multirow[t]{2}{*}{ MAR/MH } & $<50$ & Su. & $25,56.82$ & $23,52.27$ & $30,68.18$ & $34,77.27$ & $36,81.82$ & $40,90.91$ & $42,95.45$ & $26,59.09$ & $37,84.09$ \\
\hline & $>50$ & UnSu. & $19,43.18$ & $21,47.73$ & $14,31.82$ & $10,22.73$ & $8,18.18$ & $4,9.09$ & $6,13.64$ & $18,40.91$ & 7, 15.91 \\
\hline \multirow[t]{2}{*}{ PS } & $<3$ & $\mathrm{Su}$. & 33,75 & $28,63.64$ & $30,68.18$ & $31,70.45$ & $28,63.64$ & $31,70.45$ & $31,70.45$ & $32,72.73$ & $30,68.18$ \\
\hline & $>3$ & UnSu. & 11,25 & $16,36.36$ & $16,36.36$ & $13,29.55$ & $16,36.36$ & $13,29.55$ & $13,29.55$ & $12,27.27$ & $14,31.82$ \\
\hline \multirow[t]{2}{*}{ CAI1 } & - tiv & REP & $17,38.64$ & $17,38.64$ & $8,18.18$ & $17,38.64$ & $13,29.55$ & $17,38.64$ & $14,31.82$ & $14,31.82$ & 11,25 \\
\hline & + tiv & DEP & $27,61.36$ & $27,61.36$ & $36,81.82$ & $27,61.36$ & $31,70.45$ & $27,61.36$ & $30,68.18$ & $30,68.18$ & 33,75 \\
\hline \multirow[t]{2}{*}{ CAI2 } & - tiv & REP & $19,43.18$ & $17,38.64$ & $8,18.18$ & $17,38.64$ & $13,29.55$ & $17,38.64$ & $14,31.82$ & $14,31.82$ & 11,25 \\
\hline & + tiv & DEP & $25,56.82$ & $27,61.36$ & $36,81.82$ & $27,61.36$ & $31,70.45$ & $27,61.36$ & $30,68.18$ & $30,68.18$ & 33,75 \\
\hline \multirow[t]{2}{*}{ CR } & $<1$ & $\mathrm{Su}$. & $12,27.27$ & $18,40.91$ & $20,45.45$ & $23,54.27$ & $21,47.73$ & $21,47.73$ & $19,43.18$ & $20,45.45$ & $25,56.82$ \\
\hline & $>1$ & UnSu. & $32,72.73$ & $26,59.09$ & $24,54.55$ & $21,47.73$ & $23,54.27$ & $23,54.27$ & $25,56.82$ & $24,54.55$ & $19,43.18$ \\
\hline \multirow[t]{3}{*}{ TDS } & $<450$ & Best & $13,29.55$ & $12,27.27$ & $13,29.55$ & $14,31.81$ & $16,36.36$ & $14,31.81$ & $15,34.09$ & $16,36.36$ & $18,40.91$ \\
\hline & $450-2000$ & Mode & $30,68.18$ & $31,70.45$ & $30,68.18$ & $29,65.91$ & $27,61.36$ & $29,65.91$ & $28,63.64$ & $27,61.36$ & $26,59.09$ \\
\hline & $>2000$ & Hazard & $01,2.27$ & $01,2.27$ & $01,2.27$ & $01,2.27$ & $01,2.27$ & $01,2.27$ & $01,2.27$ & $01,2.27$ & 00,00 \\
\hline \multirow[t]{4}{*}{$\mathrm{TH}$} & $<75$ & Soft & $1,2.27$ & $2,4.55$ & 0,0 & 0,0 & 0,0 & 0,0 & 0,0 & 0,0 & 0,0 \\
\hline & $75-150$ & Mode & $3,6.82$ & $3,6.82$ & $6,13.64$ & $3,6.82$ & $2,4.55$ & $5,11.36$ & $3,6.82$ & $5,11.36$ & $3,6.82$ \\
\hline & $150-300$ & Hard & $23,52.27$ & $24,54.54$ & $11,25.00$ & $23,52.27$ & $21,47.73$ & $19,43.18$ & $24,6.82$ & $25,56.82$ & $24,6.82$ \\
\hline & $>300$ & V. Hard & $17,38.64$ & $15,34.09$ & $27,61.36$ & $18,40,91$ & $21,17.73$ & $20,45.45$ & $17,38.64$ & $14,31.82$ & $17,38.64$ \\
\hline \multirow[t]{2}{*}{$r_{1}$} & $<1$ & $\mathrm{Na}^{+}-\mathrm{SO}_{4}^{2-}$ & $42,95.45$ & $43,97.73$ & $41,93.18$ & $41,93.18$ & $41,93.18$ & $40,90.91$ & $43,97.73$ & $40,90.91$ & 4090.91 \\
\hline & $>1$ & $\mathrm{Na}^{+}-\mathrm{HCO}_{3}^{-}$ & $02,4.55$ & $01,2.27$ & $03,6.82$ & $03,6.82$ & $03,6.82$ & $04,9.09$ & $01,2.27$ & $04,9.09$ & $04,9.09$ \\
\hline \multirow[t]{2}{*}{$r_{2}$} & $<1$ & DMP & $38,86.36$ & $38,86.36$ & $40,90.91$ & $36,81.82$ & $41,93.18$ & $39,88.64$ & $37,84.09$ & $39,88.64$ & $34,77.27$ \\
\hline & $>1$ & SMP & $6,13.64$ & $6,13.64$ & $4,9.09$ & $08,18.18$ & $3,6.82$ & $05,11.36$ & $07,15.91$ & $05,11.36$ & $10,22.73$ \\
\hline
\end{tabular}

After "," the values are given in \%

Exc excellent, Go good, Dou doubtful, UnSu unsuitable, Perm permissible, Su suitable, REP reverse exchange process, DER direct exchange process, Mode moderate, $D M P$ deep meteoric percolation, $S M P$ shallow meteoric percolation

account rainfall effect over KR because no regular patterns were found for KR. The comparison of pre-monsoon and post-monsoon shows that KR of pre-monsoon shows more a number of wells come under suitable category after rainfall. Due to rainfall, leaching of $\mathrm{Na}^{+}$from rock to water is more in comparison with $\mathrm{Ca}^{2+}$ and $\mathrm{Mg}^{2+}$ that is why after rainfall more no. of wells come under unsuitable class (Table 5). Figure $5 \mathrm{a}, \mathrm{b}$ shows frequency of some wells under suitable and unsuitable class during the study period. KR index is alkali hazard indicator. If KR value is high, then the use of 
Table 5 Classification of fixed bore wells water during post-monsoon within the study area for irrigation based on \%Na, SAR, MH, KR RSC, TDS (Wilcox 1948; Kelly 1940; Todd 1980; USSL 1954)

\begin{tabular}{|c|c|c|c|c|c|c|c|c|c|c|}
\hline \multirow[t]{2}{*}{ Index } & \multirow[t]{2}{*}{ Range } & \multirow[t]{2}{*}{ Class } & \multicolumn{8}{|c|}{ No. of samples (with \%) under different classes per year } \\
\hline & & & 2006 & 2007 & 2008 & 2009 & 2010 & 2011 & 2012 & 2013 \\
\hline \multirow[t]{4}{*}{ SAR } & $<10$ & Exc. & $10,22.73$ & $14,31.82$ & $12,27.27$ & $15,34.09$ & $09,20.45$ & $08,18.18$ & $19,43.18$ & $20,45.45$ \\
\hline & $10-18$ & Go. & $17,22.73$ & $17,38.64$ & $12,27.27$ & $15,34.09$ & $15,34.09$ & $15,34.09$ & $13,29.55$ & $08,18.18$ \\
\hline & $18-26$ & Dou. & $08,18.18$ & $04,9.09$ & $10,22.73$ & $10,22.73$ & $10,22.73$ & $11,25.00$ & $06,13.64$ & $07,15.91$ \\
\hline & $>26$ & UnSu. & $09,20.45$ & $09,20.45$ & $10,22.73$ & $04,9.09$ & $10,22.73$ & $10,22.73$ & $06,13.64$ & $09,20.45$ \\
\hline \multirow[t]{3}{*}{ RSC/RA } & $<1.25$ & Go. & $18,40.91$ & $10,22.73$ & $10,22.73$ & $14,31.82$ & 11,25 & $20,45.45$ & $18,40.91$ & $26,59.09$ \\
\hline & $1.25-2.5$ & Dou. & $14,31.82$ & $18,40.91$ & $18,40.91$ & $20,45.45$ & 11,25 & $18,40.91$ & $17,38.64$ & $14,31.82$ \\
\hline & $>2.5$ & UnSu. & $09,20.45$ & $16,36.36$ & $16,36.36$ & $10,22.73$ & 22,50 & $06,13.64$ & $09,20.45$ & $04,9.09$ \\
\hline \multirow[t]{5}{*}{$\% \mathrm{Na}$} & $>20$ & Exce. & $01,2.27$ & 00,00 & 00,00 & 00,00 & 00,00 & 00,00 & 00,00 & $02,4.55$ \\
\hline & $20-40$ & Go. & $07,15.91$ & $09,20.45$ & $07,15.91$ & $09,20.45$ & $04,9.09$ & $05,11.36$ & $12,27.27$ & $12,27.27$ \\
\hline & $40-60$ & Perm. & $16,36.64$ & $25,56.82$ & $25,56.82$ & $23,52.27$ & $21,47.73$ & $20,45.45$ & $19,43.18$ & $15,34.09$ \\
\hline & $60-80$ & Dou. & $17,38.64$ & $10,22.73$ & $9,20.45$ & $11,25.00$ & $16,36.36$ & $16,36.36$ & $09,20.45$ & $10,22.73$ \\
\hline & $>80$ & UnSu. & $3,6.82$ & 00,00 & $03,6.82$ & $01,2.27$ & $03,6.82$ & $03,6.82$ & $04,9.09$ & $05,11.36$ \\
\hline \multirow[t]{2}{*}{$\mathrm{KR} / \mathrm{Kl}$} & $<1$ & Su. & $18,40.91$ & $28,63.64$ & 22,50 & $18,40.91$ & $16,36.36$ & $16,36.36$ & $28,63.64$ & $25,56.82$ \\
\hline & $>1$ & UnSu. & $26,59.09$ & $16,36.36$ & 22,50 & $26,59.09$ & $28,63.64$ & $28,63.64$ & $16,36.36$ & $19,43.18$ \\
\hline \multirow[t]{3}{*}{ PI } & $>75 \%$ & Su. & $05,11.36$ & $05,11.36$ & $05,11.36$ & $07,15.91$ & $05,11.36$ & $07,15.91$ & $03,6.82$ & $09,20.45$ \\
\hline & $25-75 \%$ & Go. & $39,88.64$ & $39,88.64$ & $39,88.64$ & $37,84.09$ & $38,86.36$ & 33,75 & $40,90.91$ & 33,75 \\
\hline & $<25 \%$ & UnSu. & 00,00 & 00,00 & 00,00 & 00,00 & $01,2.27$ & 00,00 & $01,2.27$ & $02,4.55$ \\
\hline \multirow[t]{2}{*}{ MAR/MH } & $<50$ & Su. & $06,13.64$ & $40,90.91$ & $06,13.64$ & $25,56.82$ & $34,77.27$ & 33,75 & 33,75 & $30,68.18$ \\
\hline & $>50$ & UnSu. & $38,86.36$ & $4,9.09$ & $38,86.36$ & $19,43.18$ & $10,22.73$ & 11,25 & 11,25 & $14,31.82$ \\
\hline \multirow[t]{2}{*}{ PS } & $<3$ & Su. & $17,38.64$ & $13,29.55$ & 11,25 & $10,22.73$ & $14,31.82$ & $14,31.82$ & $10,22.73$ & $34,77.27$ \\
\hline & $>3$ & UnSu. & $27,61.36$ & $31,70.45$ & 33,75 & $34,77.27$ & $30,68.18$ & $30,68.18$ & $34,77.27$ & $10,22.73$ \\
\hline \multirow[t]{2}{*}{ CAI1 } & - tiv & REP & $10,22.73$ & $14,31.82$ & $15,34.09$ & 11,25 & 22,50 & $3,6.82$ & $17,38.64$ & $8,18.18$ \\
\hline & + tiv & DEP & $34,77.27$ & $30,68.18$ & $29,65.91$ & 33,75 & 22,50 & $41,93.18$ & $27,61.36$ & $36,81.82$ \\
\hline \multirow[t]{2}{*}{ CAI2 } & - tiv & REP & $10,22.73$ & $14,31.82$ & $15,34.09$ & 11,25 & 22,50 & $3,6.82$ & $17,38.64$ & $8,18.18$ \\
\hline & + tiv & DEP & $34,77.27$ & $30,68.18$ & $29,65.91$ & 33,75 & 22,50 & $41,93.18$ & $27,61.36$ & $36,81.82$ \\
\hline \multirow[t]{2}{*}{ CR } & $<1$ & Su. & $16,36.36$ & $29,65.91$ & $25,56.82$ & $23,52.27$ & $24,54.55$ & $8,18.18$ & $27,61.36$ & $18,40.91$ \\
\hline & $>1$ & UnSu. & $28,63.64$ & $15,43.09$ & $19,43.18$ & $21,47.73$ & $20,45.45$ & $36,81.82$ & $17,38.64$ & $26,59.09$ \\
\hline \multirow[t]{3}{*}{ TDS } & $<450$ & Best & $12,27.27$ & 11,25 & $14,31.82$ & $14,31.82$ & $15,34.09$ & $9,20.45$ & $17,38.64$ & $18,40.91$ \\
\hline & $450-2000$ & Mode & $30,68.18$ & $31,70.45$ & $28,63.64$ & $30,68.18$ & $27,61.36$ & 33,75 & $26,59.09$ & $25,56.82$ \\
\hline & $>2000$ & Hazard & $02,4.55$ & $02,4.55$ & $02,4.55$ & 00,00 & $02,4.55$ & $02,4.55$ & $01,2.27$ & $01,2.27$ \\
\hline \multirow[t]{4}{*}{$\mathrm{TH}$} & $<75$ & Soft & 0,0 & 0,0 & 0,0 & 0,0 & 0,0 & 0,0 & 0,0 & $1,2.27$ \\
\hline & $75-150$ & Mode & $6,13.64$ & $3,6.82$ & 4, 9.09 & $5,11.36$ & $4,9.09$ & $2,4.55$ & $2,4.55$ & $8,18.18$ \\
\hline & $150-300$ & Hard & $17,38.64$ & $24,54.55$ & $20,45.45$ & $23,52.27$ & $23,52.27$ & $23,52.27$ & $25,56.82$ & $23,52.27$ \\
\hline & $>300$ & V. Hard & $21,47.73$ & $17,38.64$ & $20,45.45$ & $16,36.36$ & $17,38.64$ & $19,43.18$ & $17,38.64$ & $12,27.27$ \\
\hline \multirow[t]{2}{*}{$r_{1}$} & $<1$ & $\mathrm{Na}^{+}-\mathrm{SO}_{4}{ }^{2-}$ & $42,95.45$ & $38,86.36$ & $40,90.91$ & $41,93.18$ & $41,93.18$ & 44,100 & $42,95.45$ & $39,88.64$ \\
\hline & $>1$ & $\mathrm{Na}^{+}-\mathrm{HCO}_{3}^{-}$ & $02,4.55$ & $06,13.64$ & $04,9.09$ & $03,6.82$ & $03,6.82$ & 00,00 & $02,4.55$ & $05,11.36$ \\
\hline \multirow[t]{2}{*}{$r_{2}$} & $<1$ & DMP & $40,90.91$ & $38,86.36$ & $36,81.82$ & $41,93.18$ & $39,88.64$ & $40,90.91$ & $37,84.09$ & 39) 88.64 \\
\hline & $>1$ & SMP & $04,9.09$ & $06,13.64$ & $08,18.18$ & $03,6.82$ & $05,11.36$ & $04,9.09$ & $07,15.91$ & $05,11.36$ \\
\hline
\end{tabular}

After "," the values are given in \%

Exc excellent, Go good, Dou doubtful, UnSu unsuitable, Perm permissible, Su suitable, REP reverse exchange process, DER direct exchange process, Mode moderate, $V$ very, $D M P$ deep meteoric percolation, $S M P$ shallow meteoric percolation

pure gypsum is recommended to reduce the effect of $\mathrm{Na}^{+}$ ion.

From Tables 2 and 3, average value of PI during the study period was $59.56 \%$ and $58.75 \%$ for pre- and post-monsoon, respectively, which was under good class (25-75\%) with range from 12.10 (unsuitable, $\mathrm{PI}<25 \%$, water movement freedom is less in soil) to $99.42 \%$ (suitable, PI $>75 \%$, water movement freedom is high in soil) and 16.95 (unsuitable, $\mathrm{PI}<25 \%$ ) to $99.19 \%$ (suitable, PI $>75 \%$ ), respectively, in pre- and post-monsoon. The pre-monsoon PI of 2007 


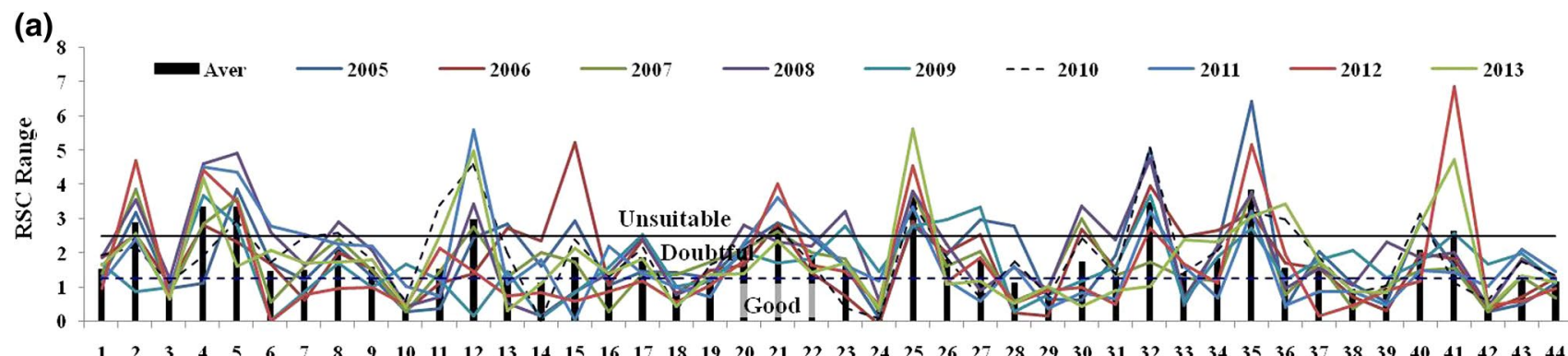

No. of Wells

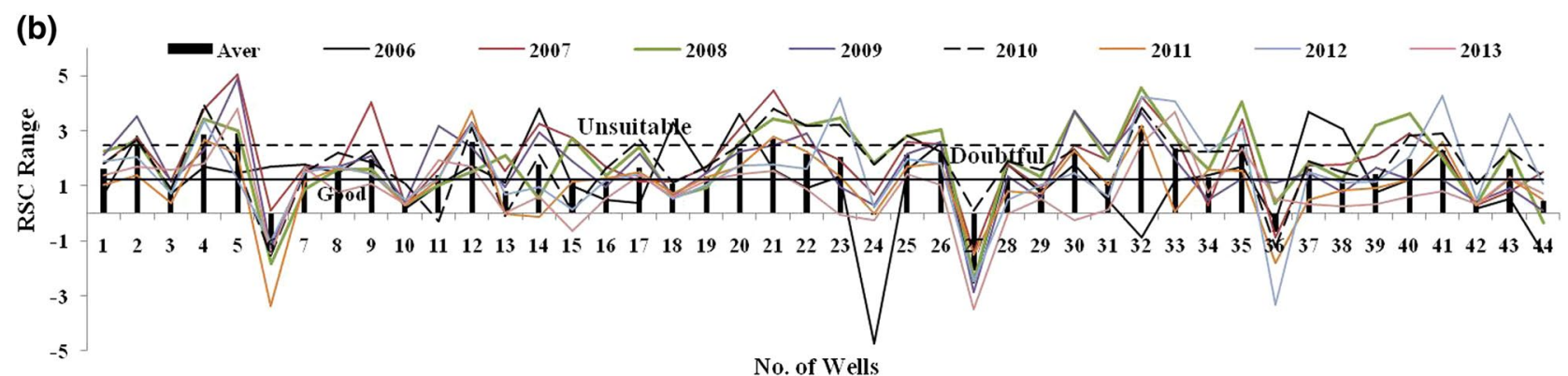

Fig. 3 a Nine-year (2005-2013) temporal representation RSC value during pre-monsoon. b Nine-year (2006-2013) temporal representation RSC value during post-monsoon

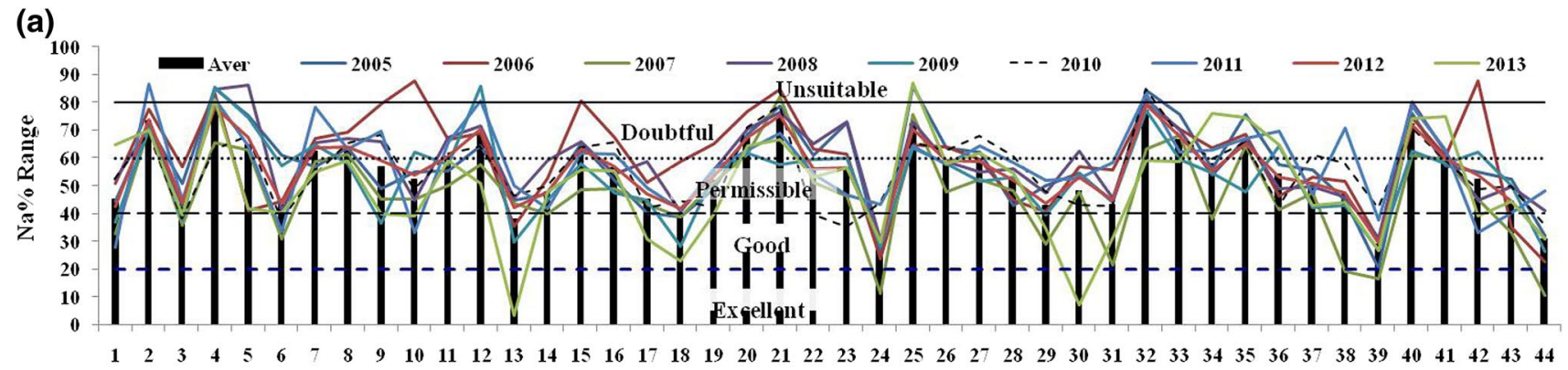
No. of Wells

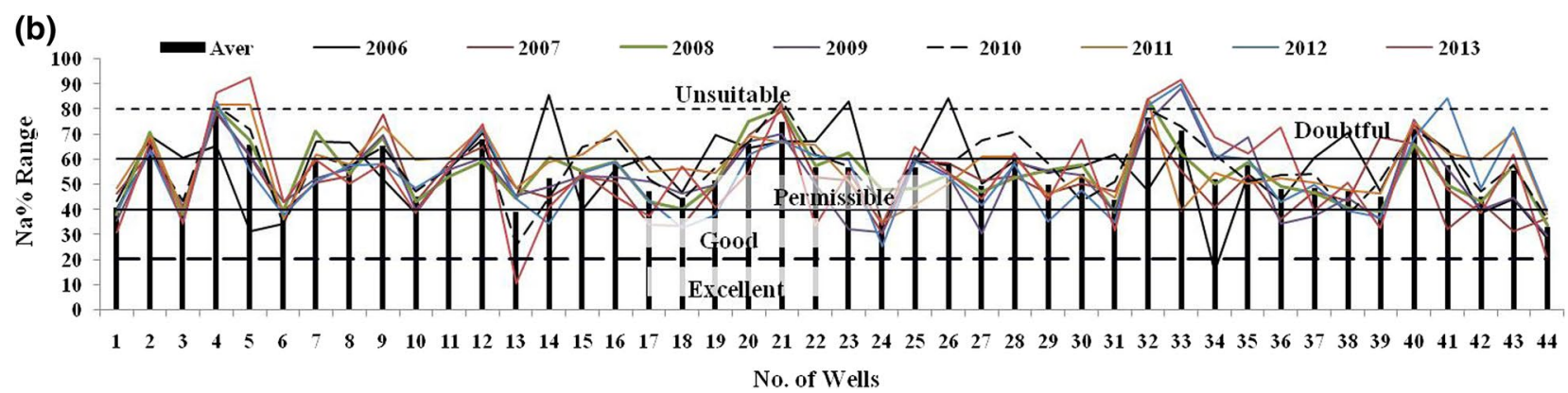

Fig. 4 a Nine-year (2005-2013) temporal representation of \% Na value during pre-monsoon. b Nine-year (2006-2013) temporal representation of $\% \mathrm{Na}$ value during post-monsoon

(Table 2) shows that the average value of PI was lowest, $51.18 \%$ (suitable); similarly, post-monsoon PI of year $2007(57.29 \%)$ and 2009 (57.08\%) was also reported low (Table 3). From Tables 2 and 3, the effect of rainfall over PI is not showing much significance. However, Tables 4 and
5 show the effect of rainfall over PI. It suggests that the no. of wells falls in unsuitable class during pre-monsoon (before rainfall) converted into suitable class after rainfall (post-monsoon). From Tables 4 and 5, it is the clear that the effect of rainfall over PI was more during the study period 
because after post-monsoon most no. of wells found under the suitable and good category. Figure $6 a$, b is capable of representing a category of particular no. of wells at the particular time of year like well nos. 12, 25 and 40 during premonsoon which come under the unsuitable category more than two times, but after post-monsoon these wells come under good class due to rainfall effect only. The soil permeability is affected by the extensive use of irrigation water as it is influenced by $\mathrm{Na}^{+}, \mathrm{Ca}^{2+}, \mathrm{Mg}^{2+}$ and $\mathrm{HCO}_{3}{ }^{-}$contents of the water (Gautam et al. 2015).

$\mathrm{MH} / \mathrm{MAR}$ is $\mathrm{Mg}^{2+}$ and $\mathrm{Ca}^{2+}$ based index, and it also represents in percent and contains only two classes $\mathrm{MH}<50 \%$ and $\mathrm{MH}>50 \%$, suitable and unsuitable, respectively. On the basis of Tables 2 and 3, average values of $\mathrm{MH}$ during the study period were 42.01 and $43.30 \%$ for pre- and post-monsoon, respectively, with range 4.46-92.98\% (pre-monsoon) and $0.85-86.44 \%$ (postmonsoon). Long-term (nine-year) average of $\mathrm{MH}$ showing majority of wells falls under suitable category of irrigation water. The average $\mathrm{MH}$ shows unsuitable limit for 2005 (pre-monsoon) and 2006 (post-monsoon) (Tables 2, 3). Table 4 shows that more than 52 percent (even $95.45 \%$ during 2011) wells in each year during pre-monsoon were in suitable $(\mathrm{MH}<50 \%)$ category, while this pattern was not shown during post-monsoon, and each year no. of wells (which come under $<50 \%$ category during premonsoon) fluctuate but never come equal or more than to no. of wells during pre-monsoon. This fluctuation during post-monsoon clearly indicates some degree of leaching process of $\mathrm{Mg}^{2+}$ (from rock to groundwater) after rainfall
(Singh et al. 2012). Figure 7a shows magnitude of $\mathrm{MH}$ for well nos. 2, 7, 12, 14, 43, 43 which were high during post-monsoon, but from Fig. 7b, after rainfall magnitude of $\mathrm{MH}$ for same wells was reduced but not below the $<50 \% \mathrm{MH}$ range. Overall, according to $\mathrm{MH}$ index $52 \%$ wells fall in suitable category of water for irrigation during pre-monsoon, but due to rainfall or $\mathrm{Mg}^{2+}$ leaching process the no. of well reduces from suitable category during postmonsoon. The year 2006 of post-monsoon elaborates that majority of wells were not unsuitable for irrigation.

If $\mathrm{MH}<50$, then it is considered as safe, and if it is greater than $>50$, then it is unsafe for irrigation use. In the analyzed groundwater samples, $61.36 \%$ of the sample lies in the unsafe $(\mathrm{MH}$ value $>50)$ and remaining $38.63 \%$ in the safe region in the pre-monsoon season. In post-monsoon season, $68.18 \%$ are unsafe and remaining $31.81 \%$ suitable for irrigation uses. According to $\mathrm{MH}$ computation, majority of groundwater samples are not suitable for irrigation purpose.

PS index (Doneen 1964) is $\mathrm{Cl}^{-}$, and $\mathrm{SO}_{4}{ }^{2-}$ dominant index. From Tables 2 and 3 (in context of PS), the long-term averages $2.52(<3)$ and $2.46(<3)$ of PS were found under suitable category during pre- and post-monsoon with much differences in minimum and maximum (12.17 and 13.26, pre- and post-monsoon, respectively). Each year's during pre- and post-monsoon, average PS value was under suitable class ( $\mathrm{PS}<3$ ). But each year (in both the monsoon) maximum value of PS was also found which was beyond of suitable limit that means few no. of wells must have come under $>3$ limit of PS. It is clear from analysis of Tables 4 and 5 , a clear signature of increasing and decreasing pattern

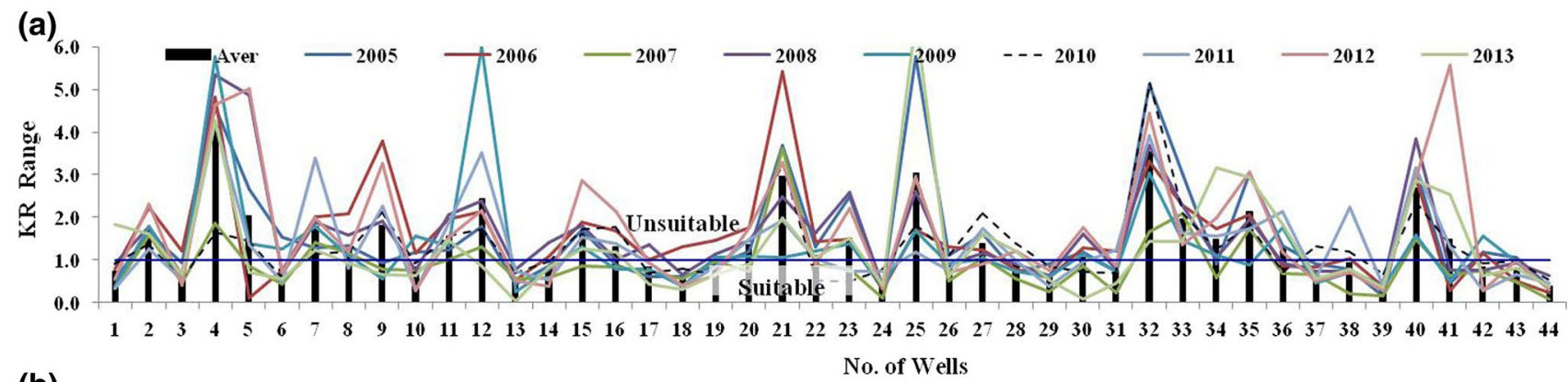

(b)

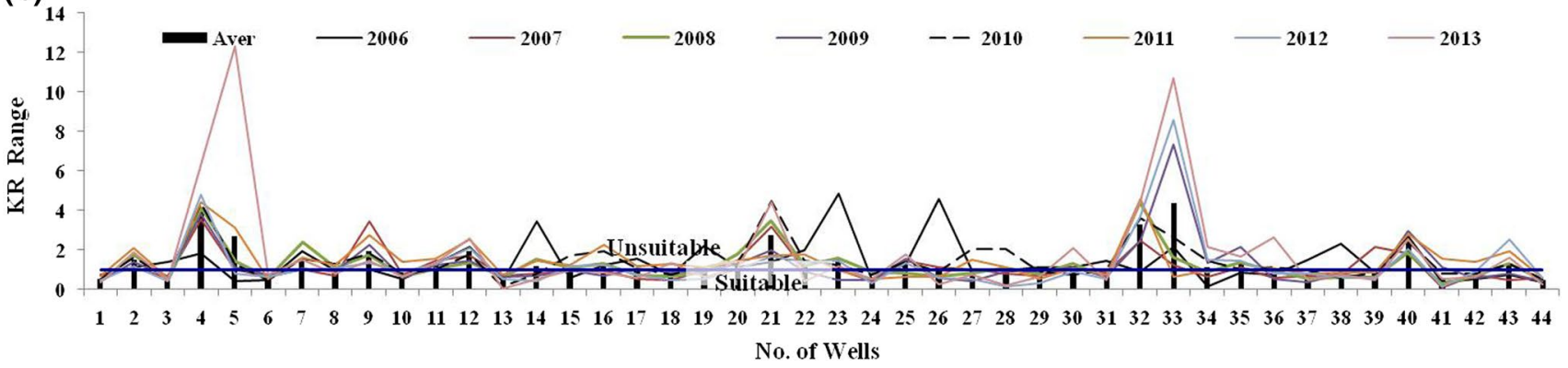

Fig. 5 a Nine-year (2005-2013) temporal representation KR/KI value during pre-monsoon. b Nine-year (2006-2013) temporal representation $\mathrm{KR} / \mathrm{KI}$ value during post-monsoon 

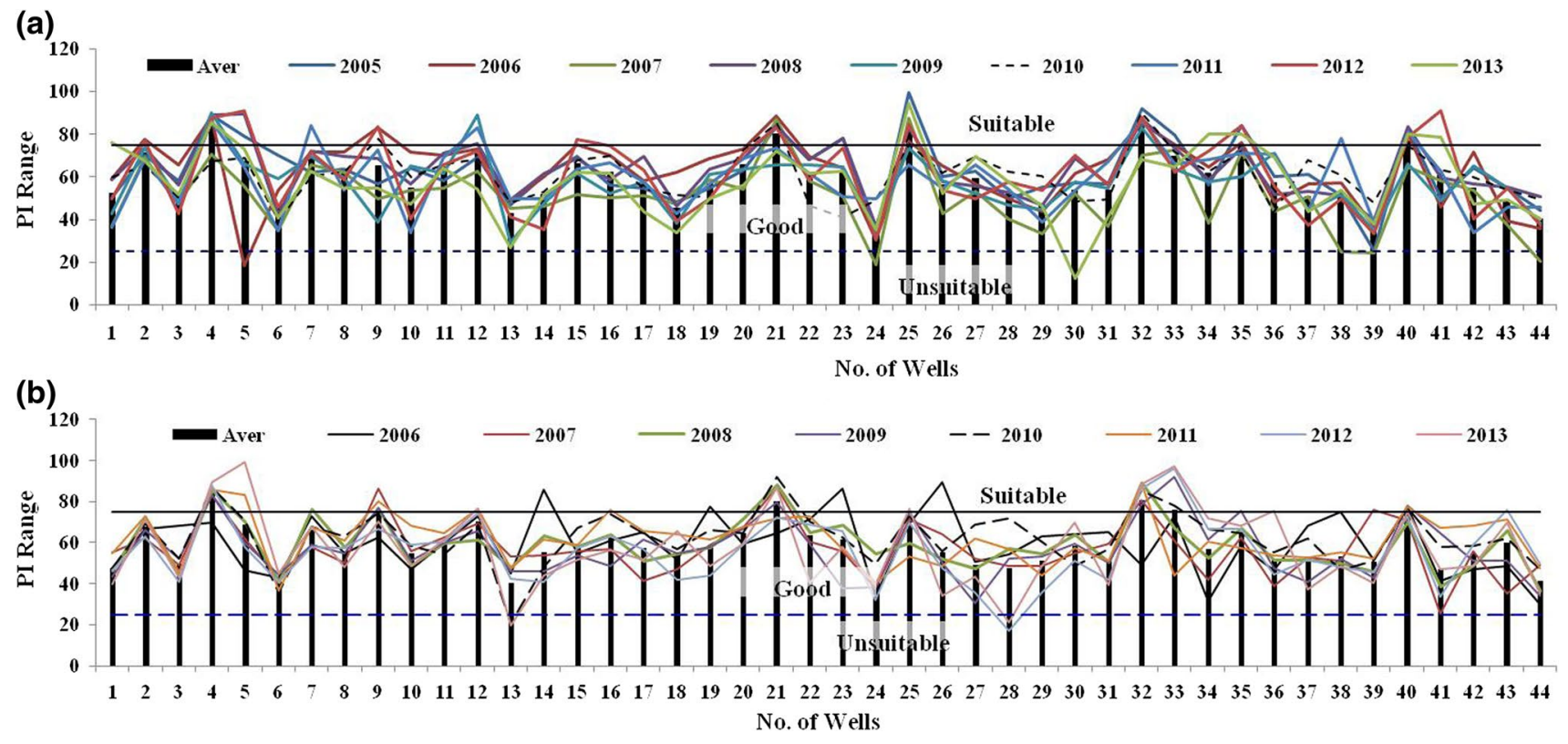

Fig. 6 a Nine-year (2005-2013) temporal representation of PI value during pre-monsoon. b Nine-year (2006-2013) temporal representation of PI value during post-monsoon

is observed in no. of wells during per- and post-monsoon, respectively. This happens due to the effect of rainfall. Figure $8 \mathrm{a}, \mathrm{b}$ shows characteristic of PS for particular no. of wells during the study time period. Well nos. $6,9,11$, $14,21,27,28,30,34,36$ and 43 have a high value of PS, but after rainfall well nos. $6,11,14,27,28,34,36$ and 43 remain constant with the unsuitable condition of water for irrigation. Overall on the basis of PS analysis, it was found that most no. of wells (more than 63.64\%) in the study area were suitable for irrigation during pre-monsoon, but during post-monsoon, more than $61.36 \%$ wells were found unsuitable for irrigation (except the year 2013).

The CAI1 and CAI2 indexes indicate the direction of reaction (DRP or ERP) between groundwater and aquifer.

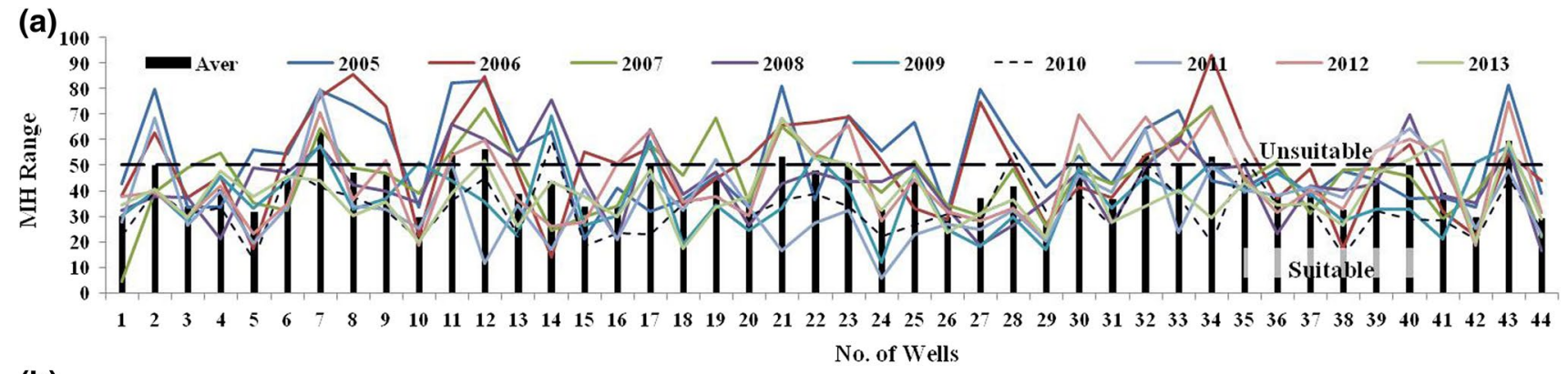

(b)

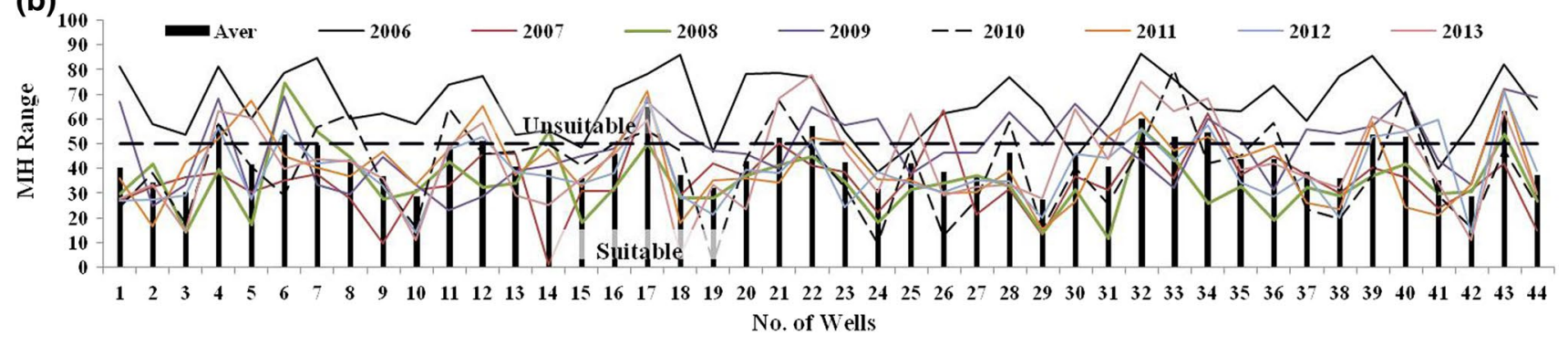

Fig. 7 a Nine-year (2005-2013) temporal representation of MH value during pre-monsoon. b Nine-year (2006-2013) temporal representation of $\mathrm{MH}$ value during pre-monsoon 
From Tables 2 and 3 (in references of CAI1 and CAI2), according to long-term (nine-year) average value during preand post-monsoon CAI1 (0.02 and 0.04) and CAI2 (0.19 and 0.27 ), it was found that most of the year DRP happens between groundwater and aquifer in the study area over the study period. From Tables 4 and 5, it is clear that during the study period most no. of wells (more than 61.36 percent and max. $81.82 \%$ ) come under DRP condition except the year 2010 during post-monsoon, 50\% show DRP, and remaining 50\% show ERP. Almost equal no. of wells were same for CAI1 and CAI 2 during pre- and post-monsoon; therefore, CAI1 and CAI2 indexes show supporting nature to each other. Figures $9 \mathrm{a}, \mathrm{b}$ and $10 \mathrm{a}, \mathrm{b}$ reveal the supporting nature, magnitudes may be not same, but naturewise [negative (-, ERP) and positive $(+$, DRP)], they are same.

$\mathrm{CR}$ is $\mathrm{Cl}^{-}$dominant index similar to PS, CAI1 and CAI2. Hence, it suggests the same nature of water. Well nos. 6, 12, $13,14,15,24,27,36$ and 44 show almost same increasing nature of these indexes (Figs. 8a, d, 9a, b, 10a, b, 11a, b), but magnitude is different). CR can be used as an economical index in point of irrigation supply system because on the basis of CR it can be decided that irrigation water could be transported by PVC plastic (low cost) pipes or metallic pipes (cost effective). Knowledge of CR index is crucial when irrigation drainage is long. When irrigation water is low in open drainage system, there is a chance of contamination. The supply of irrigation water through the pipes is recommended (may be metallic or PVC plastic pipes) on the basis of CR values of irrigation water.
The fluctuations in TDS are shown in Fig. 12a, b while its statistics is given in Tables 2, 3. According to Tables 2 and 3 , average value of TDS was found 749.94 and $729.14 \mathrm{mg} / \mathrm{l}$ for pre- and post-monsoon, respectively. While during both seasons average values fall under the moderate category $(450-2000 \mathrm{mg} / \mathrm{l})$. Tables 4 and 5 give the information about TDS fluctuation binwise. From Table 4, maximum no. of wells fall under best (TDS $<450 \mathrm{mg} / \mathrm{l}$ ) and moderate category, while each year during pre-monsoon one well comes under hazard category (> $2000 \mathrm{mg} / \mathrm{l}$, Table 4). Similarly, during post-monsoon most no. of wells distributed under best and the moderate bin (Table 4), while each year one or two wells come under hazard category. Tables 4 and 5 clearly reveal that during pre- and post-monsoon most no. of wells come under moderate class only. From Fig. 12a, b, it is clear that particular well no. 27 comes under hazard category during both seasons and almost same time of year (2006, 2007, 2008, 2010, 2011 and 2013), while well nos. 24,34 and 36 fall under hazard category during postmonsoon which may be increasing salt concentration after rainfall due to weathering of salt from rocks. On the basis of TDS analysis, we found all wells water was suitable for irrigation (except well no. 27) during pre-monsoon, but after rainfall some salt concentration increases which influences TDS value of some wells; therefore, some treatment is required in particular wells water before using water for irrigation.

Water hardness is represented by TH, and it is $\mathrm{Ca}^{2+}$ and $\mathrm{Mg}^{2+}$ dominate index. From Tables 2 and 3 (in context of
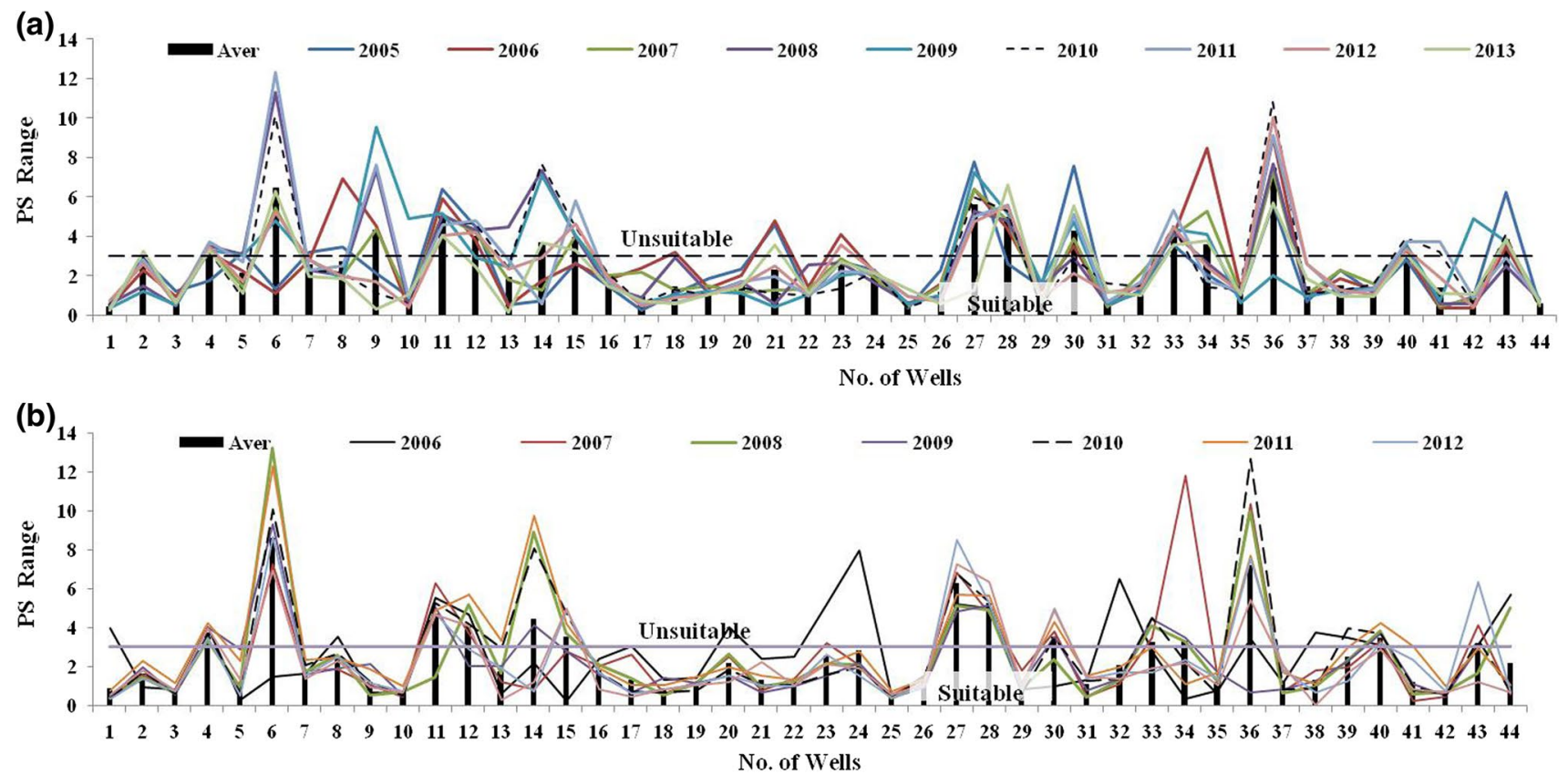

Fig. 8 a Nine-year (2005-2013) temporal representation of PS value during pre-monsoon. b Nine-year (2006-2013) temporal representation of PS value during post-monsoon 


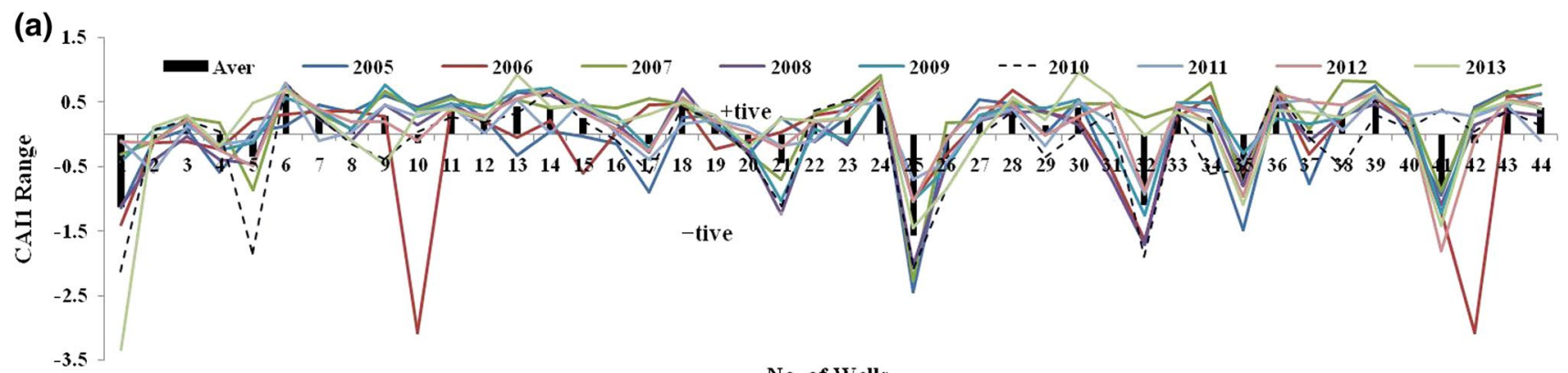

No. of Wells

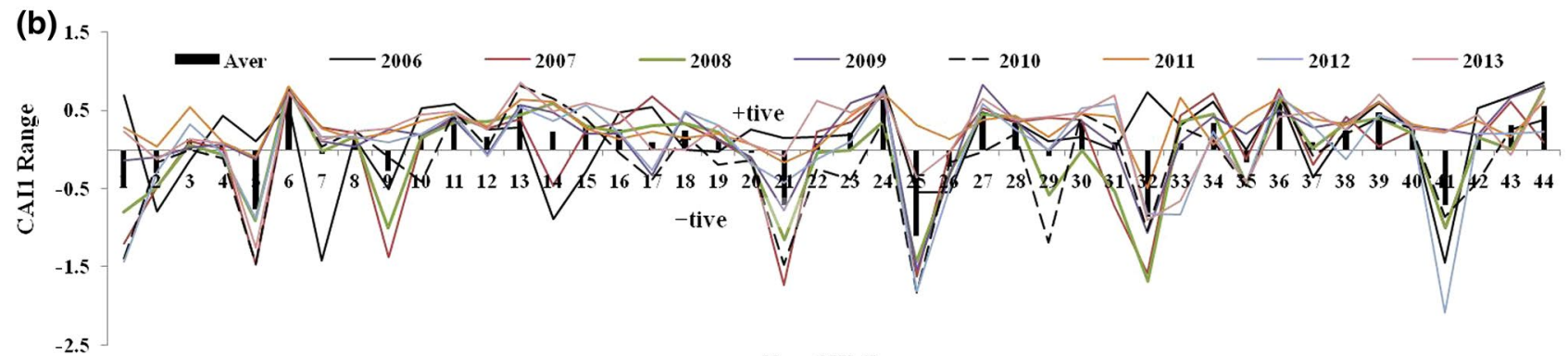

No. of Wells

Fig. 9 a Nine-year (2005-2013) temporal representation of CIA1 value during pre-monsoon. b Nine-year (2006-2013) temporal representation of CAI1 value during post-monsoon
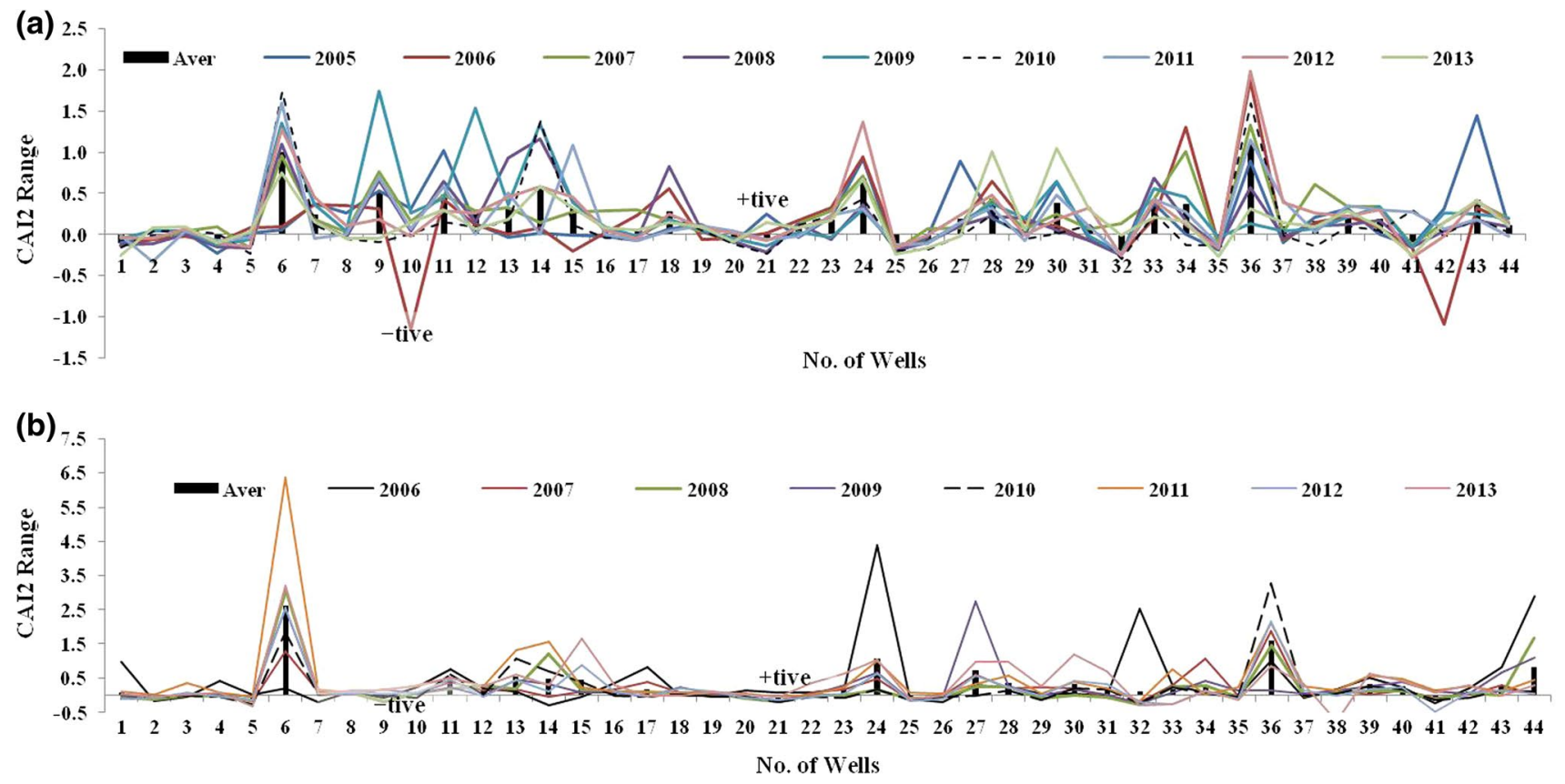

Fig. 10 a Nine-year (2005-2013) temporal representation of CAI2 value during pre-monsoon. b Nine-year (2006-2013) temporal representation of CAI2 value during post-monsoon

$\mathrm{TH})$, we found that average value of $\mathrm{TH}$ for study time period was 385.21 and $356.02 \mathrm{mg} / \mathrm{l}$ during pre- and post-monsoon, average value of $\mathrm{TH}$ shows that most no. wells in the study area were very hard $(\mathrm{TH}>300 \mathrm{mg} / \mathrm{l})$ condition for long time period. However, TH ranged from 51 to $1495.60 \mathrm{mg} / \mathrm{l}$
(Table 2) and 69.82-1646.40 mg/l (Table 3) during pre- and post-monsoon, respectively, and has revealed that maximum TH limit of 1495.60 (pre-monsoon) and 1646.40 (post-monsoon) $\mathrm{mg} / \mathrm{l}$ were so high from $\mathrm{TH}>300 \mathrm{mg} / \mathrm{l}$ (hazard condition). However, Tables 4 and 5 present the number of wells 

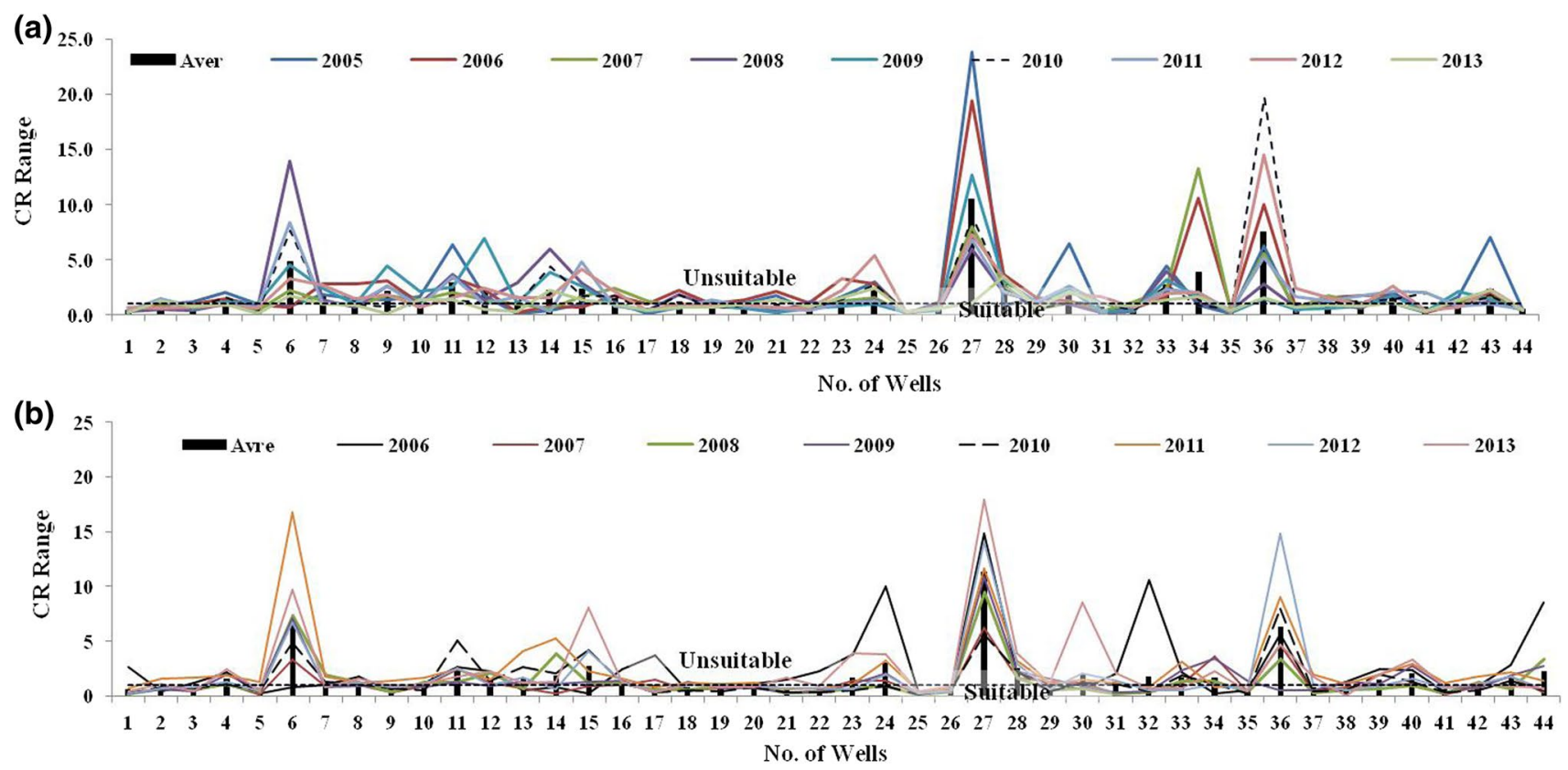

Fig. 11 a Nine-year (2005-2013) temporal representation of CR value during pre-monsoon. b Nine-year (2006-2013) temporal representation of CR value during post-monsoon

and their class during the study period. Table 4 shows that during the study period (pre-monsoon) the study area has faced a shortage of soft and moderate classes of water for irrigation. On the basis of Table 4, no. of wells are classified into two classes as hard (150-300 mg/l) and very hard
(TH>300 mg/l). Few wells fall in moderate categories, and in soft categories, no well falls. Similarly, during post-monsoon, most no. of wells come under two classes, hard and very hard (Table 5). Figure 13a, b, shows characteristic of each well during pre- and post-monsoon. Figure 13a reveals
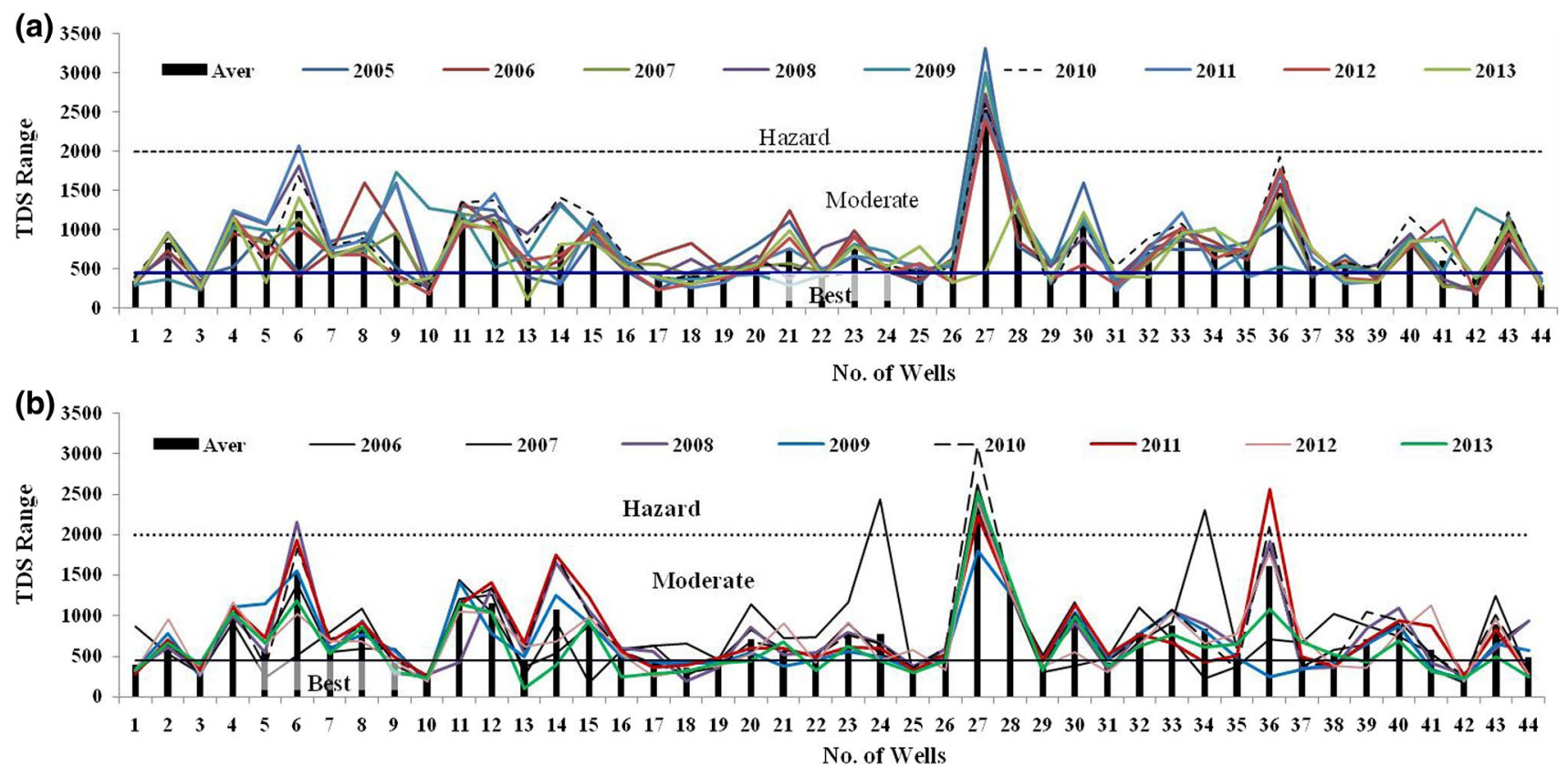

Fig. 12 a Nine-year (2005-2013) temporal representation of TDS value during pre-monsoon. b Nine-year (2006-2013) temporal representation of TDS value during post-monsoon 

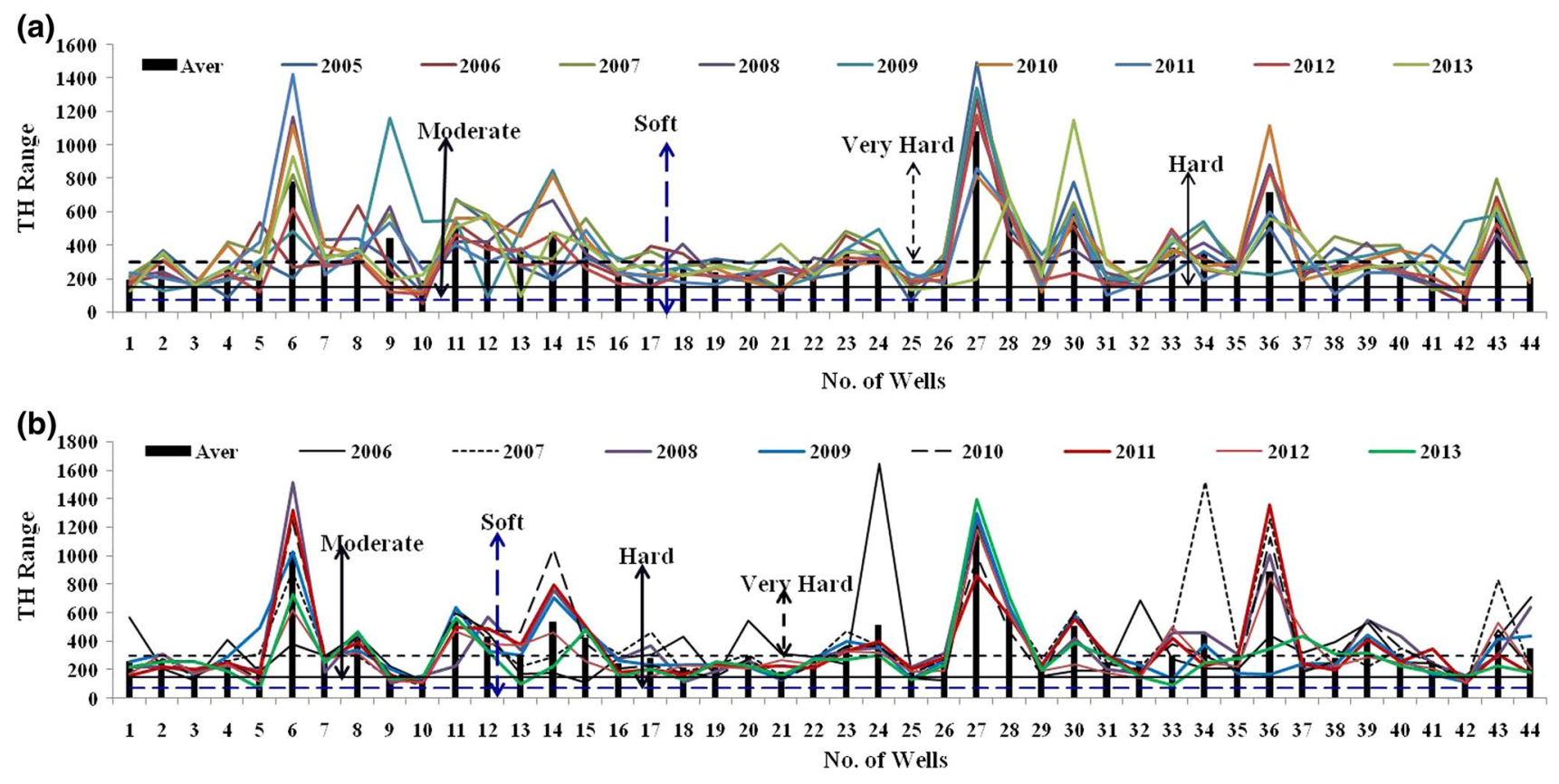

Fig. 13 a Nine-year (2005-2013) temporal representation of TH value during pre-monsoon. b Nine-year (2006-2013) temporal representation of TH value during post-monsoon
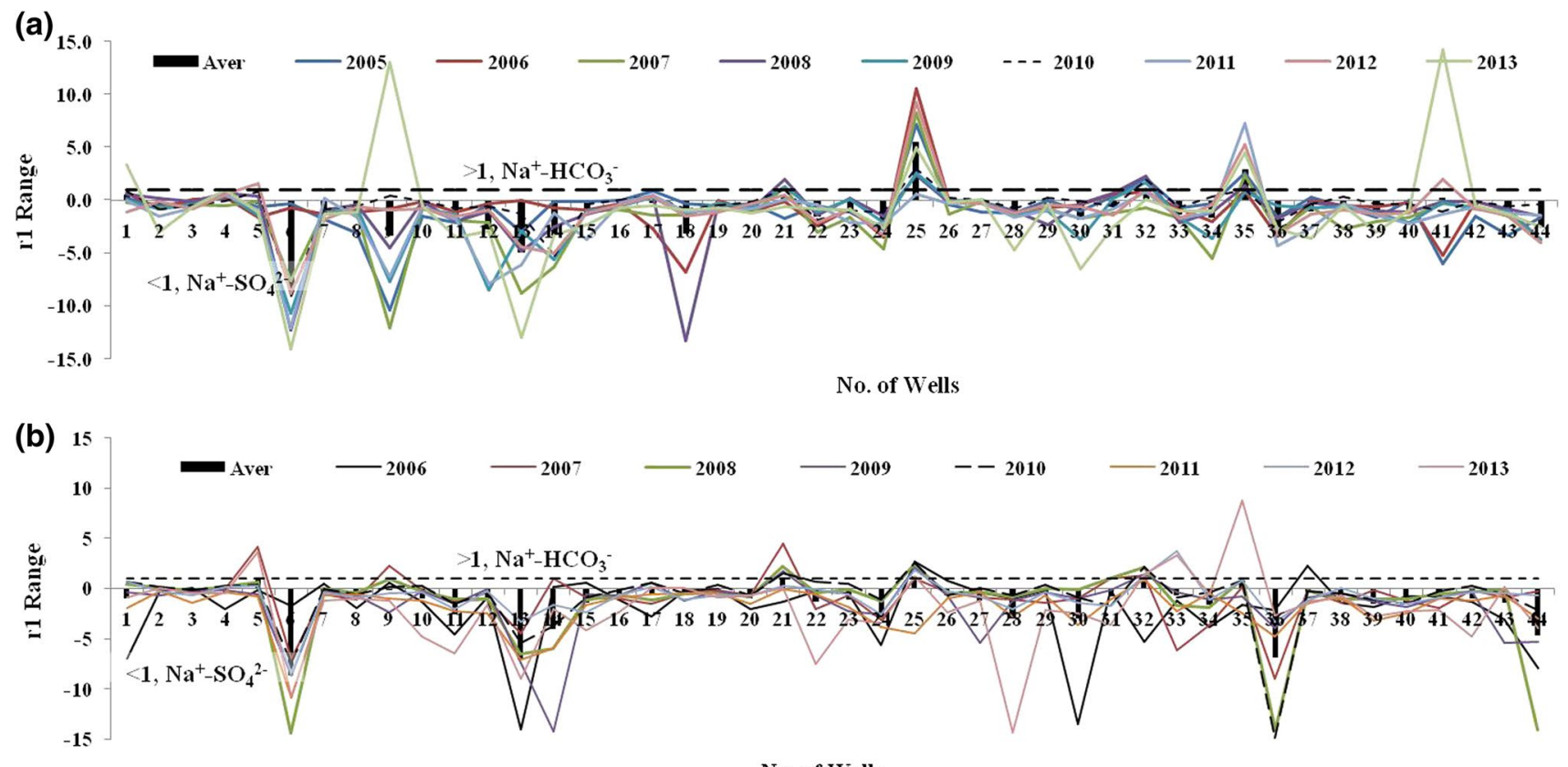

No. of Wells

Fig. 14 a Nine-year (2005-2013) temporal representation of $r_{1}$ value during pre-monsoon. b Nine-year (2006-2013) temporal representation of $r_{1}$ value during post-monsoon

that the well nos. 6, 14, 27 30, 36 and 43 show abnormal jump from $300 \mathrm{mg} / \mathrm{l}$ with respect to other wells during premonsoon. Similarly, Fig. 13b shows the same thing for well nos. 6, 14, 27, 36 and 43 during post-monsoon. Overall rainfall not shows any effect over TH.
Finally, industrial fixed wells are discriminated on the basis of Soltan (1998) index $r_{1}$. Index $r_{1}$ suggests water type $\left(\mathrm{Na}^{+}-\mathrm{HCO}_{3}{ }^{-}\right.$and $\left.\mathrm{Na}^{+}-\mathrm{SO}_{4}{ }^{2-}\right)$. From Tables 2 and $3\left(r_{1}\right)$, during study time period average water type was $\mathrm{Na}^{+}-\mathrm{SO}_{4}{ }^{2-}$ because the average value of $r_{1}$ belongs to less 


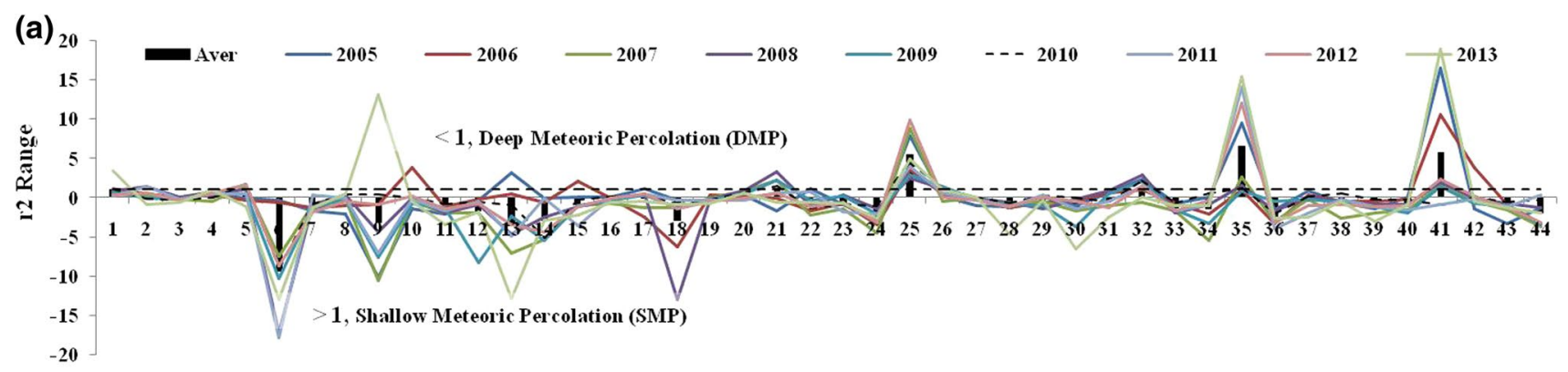

No. of Wells

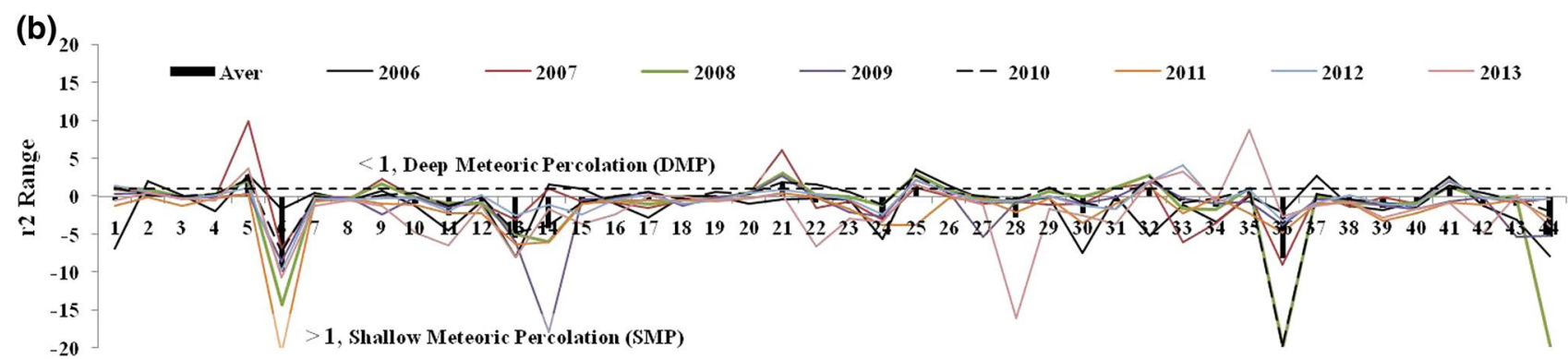

No. of Wells

Fig. 15 a Nine-year (2005-2013) temporal representation $r_{2}$ value during pre-monsoon. b Nine-year (2006-2013) temporal representation $r_{2}$ value during post-monsoon

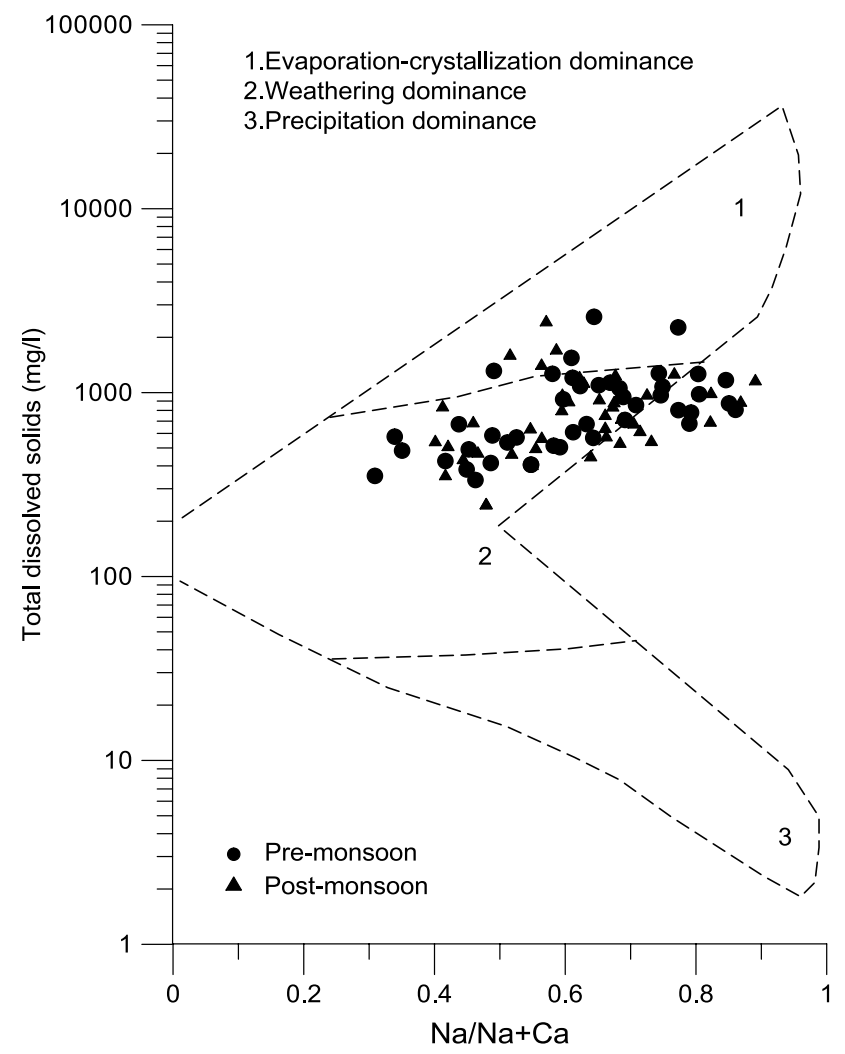

Fig. 16 Gibbs diagram shows that in both the pre-monsoon and postmonsoon seasons water-rock/soil interaction is responsible for the chemical composition of the groundwater in the study area

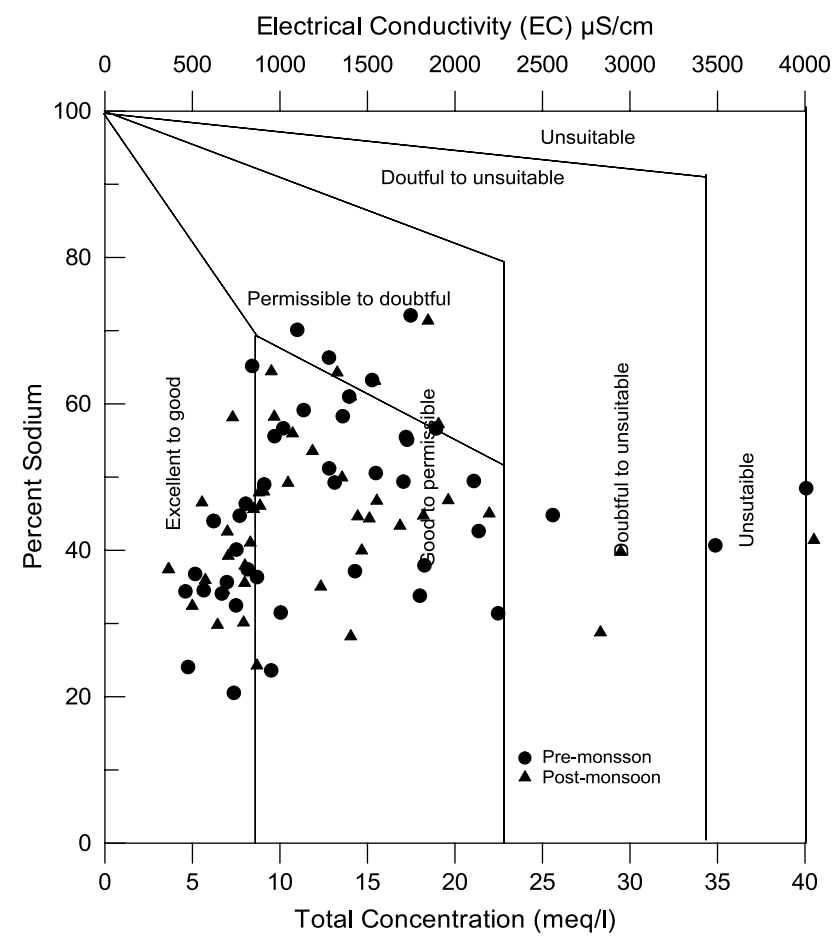

Fig. 17 Wilcox diagram shows the water suitability for irrigation use

than 1 ( -1.12 and -1.46 , pre- and post-monsoon, respectively). In yearwise average, water type was also found as $\mathrm{Na}^{+}-\mathrm{SO}_{4}{ }^{2-}$ (in both sessions); however, few $r_{1}$ value was reported greater than 1 (because maximum value of $r_{1}$ during 


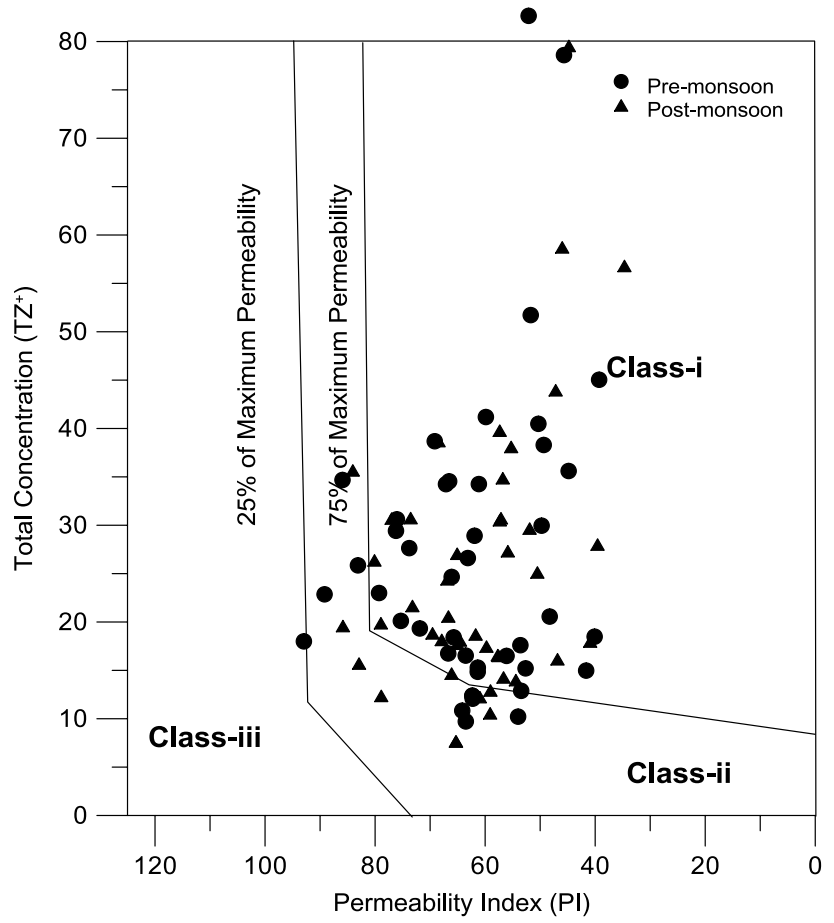

Fig. 18 Doneen (1964) classified irrigation water based on the permeability index

pre- and post-monsoon was 14.25 and 8.80 , respectively), and water type is $\mathrm{Na}^{+}-\mathrm{HCO}_{3}{ }^{-}$. Figure $14 \mathrm{a}, \mathrm{b}$ shows that water type did not change. Only magnitude of $r_{1}$ changed during pre- and post-monsoon; therefore, rainfall effect came over the magnitude of $r_{1}$ but not the type of water.

Soltan (1998) also suggested another index $\left(r_{2}\right)$ for classification of water system based on the yield of $\mathrm{K}^{+}, \mathrm{Na}^{+}$, $\mathrm{Cl}^{-}$and $\mathrm{SO}_{4}{ }^{2-}$ ions in water. On the basis of $r_{2}$, no. of well water was categorized as DMP $\left(r_{2}<1\right)$ and SMP $\left(r_{2}>1\right)$; according to average value of $r_{2}(-0.59$ and -1.17 , pre- and post-monsoon, respectively) during the study period, the no. of well's water was categorized as DMP (Tables 2, 3, in context of $r_{2}$ ). Whoever some wells (max 18\%) show SMP nature also (Tables 4, 5). From Fig. 15a, b, only the magnitude of $r_{2}$ was fluctuated not nature of water (DMP/SMP).

The Gibbs diagram (Gibbs 1970; Fig. 16) is a commonly used method to establish the relationship of water composition and aquifer lithological characteristics. Three distinct fields of the evaporation-crystallization, weathering/rock-water interaction and precipitation dominance are shown in the boomerang-shaped Gibbs diagram. The distribution of the sampling points elucidates that the major ion chemistry of the groundwater seems to be controlled by chemical weathering of rock forming minerals and anthropogenic activities (Singh et al. 2012; Gautam et al. 2015).

The Wilcox (1948) diagram elucidates sodium percentage and electrical conductivity to classify groundwater (Fig. 17), which can be divided into five divisions (excellent to good, good to permissible, permissible to doubtful, doubtful to unsuitable and unsuitable). As per the Bureau of Indian Standard (BIS), maximum sodium of $60 \%$ is recommended for irrigation water (Gautam et al. 2015). Doneen (1964) classified irrigation water based on the permeability index (Fig. 18). The soil permeability is affected by the extensive use of irrigation water as it is influenced by $\mathrm{Na}^{+}, \mathrm{Ca}^{2+}, \mathrm{Mg}^{2+}$ and $\mathrm{HCO}_{3}{ }^{-}$content of the water (Gautam et al. 2015).

\section{Conclusion}

The study area is under stress due to rock element reactions with groundwater (and influences geochemistry of groundwater), and some effects of land-use change (especially where the aquifer is unconfined) are due to urbanization, sea (saline) water interference (study area near of sea) and aquifer refreshing, and over-exploitation. The hydro-geochemical index-based study reveals that the groundwater is hard to very hard. The groundwater of the study area is more influenced by rock-water interaction, sea water, dominance and evaporation (due to high temperature) dominance field. The $r_{1}$ and $r_{2}$ indexes show that groundwater is $\mathrm{Na}^{+}-\mathrm{SO}_{4}{ }^{2-}$ and deep meteoric percolation (DMP) type. Based on the indexes [SAR, SSP (or $\% \mathrm{Na}$ ), RSC (or RA), KR, PI, CAI, PS, MH, TDS and TH] groundwater majority of the wells is falling under moderate to the unsuitable category of water for irrigation purposes. Thus, the use of groundwater for irrigation (without treatment) in the study area will damage crops and reduces production. However, more saline or salt-tolerable crops could be grown with a good irrigation system to stop soil salinization [also use lime/gypsum treatment to bout permeability of the soils (base-exchange process)]. The outcomes of this study show that index-based irrigation water quality can be useful in decision-making processes such as identifying no. of suitable wells for irrigation systems and prevent the damage of crops and production. It is also suggested that regular monitoring of irrigation water quality and pollution is essential to help farmers and the concerned department for making irrigation policy to the state government. This would be helpful in developing the suitable management plan and sustainable utilization of groundwater in irrigation. To recover the good quality of groundwater, the Government, semi-government and nongovernment organization (NGO) should make available and support to design the artificial recharge structures and rainwater harvesting structures in the study area.

Acknowledgements The authors are grateful to K. Santhanam [Former Joint Director (Geology), Water Resources Division, P.W.D, Chennai], for providing water quality datasets and thank Dr. Anil Kumar Mishra 
(Principal Scientist, Water Technology Centre, IARI, New Delhi) for his critical input and suggestions on the manuscript. SKG expresses sincere thanks to the Science and Engineering Research Board (SERB), New Delhi, India, for providing the financial support DST N-PDF (File No: PDF/2017/002820).

Open Access This article is distributed under the terms of the Creative Commons Attribution 4.0 International License (http://creativeco mmons.org/licenses/by/4.0/), which permits unrestricted use, distribution, and reproduction in any medium, provided you give appropriate credit to the original author(s) and the source, provide a link to the Creative Commons license, and indicate if changes were made.

\section{References}

Al-Shammiri M, Al-Saffar A, Bohamad S, Ahmed M (2005) Waste water quality and reuse in irrigation in kuwait using microfiltration technology in treatment. Desalination 185(1-3):213-225

Amin A, Fazal S, Mujtaba A, Singh SK (2014) Effects of land transformation on water quality of Dal lake, Srinagar, India. J Indian Soc Remote Sens 42:119-128

Aravindan S (2004) Groundwater quality in the hard rock area of the Gadilam River Basin, Tamilnadu. Tour Geo Soc 63:625-635

Ayers RS, Westcot DW (1985) Water quality for agriculture. Irrigation and drainage paper No. 29. Food and agriculture organization of the United Nations, Rome, pp 1-117

Balasubramanian A (1986) Hydrogeological investigation of Tambraparani River Basin, Tamilnadu. Unpublished PhD thesis, University of Mysore, p 349

Collins R, Jenkins A (1996) The impact of agricultural land use on stream chemistry in the middle hills of the Himalayas, Nepal. J Hydrol 185:71-86

Das A, Munoz-Arriola F, Singh SK, Jha PK, Kumar M (2017) Nutrient dynamics of the Brahmaputra (Tropical River) during the monsoon period. Desalin Water Treat Sci Eng 76:212-224

Doneen LD (1964) Notes on water quality in agriculture. Published as a water science and engineering paper 4001, Department of Water Science and Engineering, University of California, Davis

Eaton FM (1950) Significance of carbonate in irrigation water. Soil Sci 69(2):123-133

FAO (Food and Agriculture Organization) (2006) Prospects for food, nutrition, agriculture, and major commodity groups. World agriculture: toward 2030-2050, Interim report. Global Perspective Studies Unit. FAO, Rome

Gajbhiye S, Singh SK, Sharma SK (2015) Assessing the effects of different land use on water qualify using multi-temporal landsat data. In: Siddiqui AR, Singh PK (eds) Resource management and development strategies: a geographical perspective. Pravalika Publication, Allahabad, pp 337-348. ISBN: 91 8-93-84292-21-8

Gautam SK, Sharma D, Tripathi JK, Ahirwar S, Singh SK (2013) A study of the effectiveness of sewage treatment plants in Delhi region. Appl Water Sci 3:57-65. https://doi.org/10.1007/s1320 1-012-0059-9

Gautam SK, Maharana C, Sharma D, Singh AK, Tripathi JK, Singh SK (2015) Evaluation of groundwater quality in the Chotanagpur Plateau region of the Subarnarekha River Basin, Jharkhand State, India. Sustain Water Qual Ecol 6:57-74. https://doi.org/10.1016/j. swaqe.2015.06.001

Gautam SK, Singh AK, Tripathi JK, Singh SK, Srivastava PK, Narsimlu B, Singh P (2016) Appraisal of surface and groundwater of the Subarnarekha River Basin, Jharkhand, India: using remote sensing, irrigation indices and statistical techniques. In: Srivastva
PK, Pandey PC, Kumar P, Raghubanshi ASHD (eds) Geospatial technology for water resource applications. CRC Press, Boca Raton, pp 144-169

Gautam SK, Tziritis E, Singh SK, Tripathi JK, Singh AK (2018) Environmental monitoring of water resources with the use of PoS index: a case study from Subarnarekha River basin, India. Environ Earth Sci 77:70. https://doi.org/10.1007/s12665-018-7245-5

Gibbs RJ (1970) Mechanism controlling world water chemistry. Science 170:795-840

Gowd SS (2005) Assessment of groundwater quality for drinking and irrigation purposes: a case study of Peddavanka watershed, Anantapur District, Andhra Pradesh, India. Environ Geol 48(6):702-712

Gupta P, Vishwakarma M, Rawtani PM (2009) Assessment of water quality parameters of Kerwa Dam for drinking suitability. Int J Theor Appl Sci 1(2):53-55

Jacintha TGA, Rawat KS, Mishra A, Singh SK (2016) Hydrogeochemical characterization of groundwater of Penninsular Indian region using multivariate statistical techniques. Appl Water Sci. https:// doi.org/10.1007/s13201-016-0400-9

Joshi DM, Kumar A, Agrawal N (2009) Assessment of the irrigation water quality of River Ganga in Haridwar District India. J Chem 2(2):285-292

Karanth KR (1987) Groundwater assessment development and management. Tata McGraw Hill Publishing Company Ltd., New Delhi, p 725

Keesari T, Ramakumar KL, Chidambaram S, Pethperumal S, Thilagavathi R (2016a) Understanding the hydrochemical behavior of groundwater and its suitability for drinking and agricultural purposes in Pondicherry area, South India - a step towards sustainable development. Groundw Sustain Dev 2-3:143-153. https:// doi.org/10.1016/j.gsd.2016.08.001

Keesari T, Sinha UK, Deodhar A, Krishna SH, Ansari A, Mohokar H (2016b) High fluoride in groundwater of an industrialized area of Eastern India (Odisha): inferences from geochemical and isotopic investigation. Environ Earth Sci 75:1090. https://doi.org/10.1007/ s12665-016-5874-0

Kelly WP (1940) Permissible composition and concentration of irrigated waters. In: Proceedings of the A.S.C.F, 607

Khodapanah L, Sulaiman WNA, Khodapanah DN (2009) Groundwater quality assessment for different purposes in Eshtehard District, Tehran, Iran. Eur J Sci Res 36(4):543-553

Milovanovic M (2007) Water quality assessment and determination of pollution sources along the Axios/Vardar River, Southeast Europe. Desalination 213:159-173

Modi PN (2000) Water engineering and water power engineering. New Delhi Press, New Delhi, pp 339-360

Narsimha A, Sudarshan V, Swathi P (2013) Groundwater and its assessment for irrigation purpose in Hanmakonda Area, Warangal District, Andhra Pradesh, India. Int J Res Chem Environ 3:195-199

Nemčić-Jurec J, Singh SK, Jazbec A, Gautam SK, Kovac I (2017) Hydrochemical investigations of groundwater quality for drinking and irrigational purposes: two case studies of Koprivnica-Križevci County (Croatia) and district Allahabad (India). Sustain Water Resour Manag. https://doi.org/10.1007/s40899-017-0200-x

Paliwal KV (1967) Effect of gypsum application on the quality of irrigation water. Madras Agric J 59:646-647

Raghunath HM (1987) Ground water, 2nd edn. Wiley, New Delhi, p 353

Ramesh K, Elango L (2012) Groundwater quality and its suitability for domestic and agricultural use in Tondiar River Basin, Tamil Nadu, India. Environ Monit Assess 184(6):3887-3899

Rawat KS, Singh SK (2018) Water quality indices and GIS-based evaluation of a decadal groundwater quality. Geol Ecol Landsc. https://doi.org/10.1080/24749508.2018.1452462

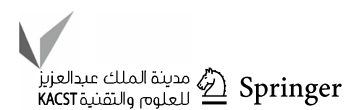


Rawat KS, Tripathi VK (2016) Standardized precipitation index based approach for development of regional drought monitoring system. J Remote Sens Technol 4(1):48-57

Rawat KS, Mishra AK, Paul G, Kumar R (2012a) Estimation of ground water recharge in Shankergarh block of Allahabad (India) using remote sensing data and statistical approach. Glob J Sci Eng Technol 2(1):34-48

Rawat KS, Mishra AK, Sehgal VK (2012b) Identification of geospatial variability of fluoride contamination in ground water of Mathura District, Uttar Pradesh, India. J Appl Nat Sci 4(1):117-122

Rawat KS, Mishra AK, Sehgal VK, Tripathi VK (2012c) Spatial variability of ground water quality in Mathura District (Uttar Pradesh, India) with geostatistical method. Int J Remote Sens Appl 2(1):1-9

Rawat KS, Mishra AK, Sehgal VK (2012d) Spatial variability of satellite derived Rainfall erosivity factors (R-Factors) for a watershed near Allahabad. J Agric Phys 11(1):71-78

Rawat KS, Tripathi VK, Kala PM, Singh M (2016) Groundwater recharge assessment using remote sensing. In: NCWES-2016 (Proceeding), Centre for Water Resources Institute of Science and Technology JNTU Hyderabad Kukatpally, Hyderabad, vol 85, pp 385-377

Rawat KS, Tripathi VK, Singh SK (2017) Groundwater quality evaluation using numerical indices: a case study (Delhi, India). Sustain Water Resour Manag. https://doi.org/10.1007/s40899-017-0181-9

Rawat KS, Singh SK, Jacintha TGA, Nemčić-Jurec J, Tripathi VK (2018) Appraisal of long term groundwater quality of peninsular India using water quality index and fractal dimension. J Earth Syst Sci. https://doi.org/10.1007/s12040-017-0895-y

Richard LA (1954) Diagnosis and improvement of saline alkali soils. US Department of Agriculture, Hand book no. 60, p 160

Rowe DR, Abdel-Magid IM (1995) Handbook of wastewater reclamation and reuse. CRC Press, Boca Raton

Saleh A, Srinivasula SM, Acharya S, Fishel R, Alnemri ES (1999) Cytochrome $\mathrm{c}$ and dATP-mediated oligomerization of apaf-1 is a prerequisite for procaspase- 9 activation. $\mathrm{J}$ Biol Chem 274:17941-17945

Sastri JCV (1994) Groundwater chemical quality in river basins, hydrogeochemical modeling. Lecture notes: refresher course. School of Earth Sciences. Bharathidasan University, Tiruchirapalli, Tamil Nadu, India

Schoeller H (1977) Geochemistry of groundwater. In: Groundwater studies - an international guide for research and practice, vol 15. UNESCO, Paris, pp 1-18

Shankar K, Aravindan S, Rajendran S (2011) Hydrogeochemistry of the Paravanar River Sub-basin, Cuddalore District, Tamil Nadu. E-J Chem 8(2):835-845
Singh S, Singh C, Kumar K, Gupta R, Mukherjee S (2009) Spatialtemporal monitoring of groundwater using multivariate statistical techniques in Bareilly district of Uttar Pradesh, India. J Hydrol Hydromech 57:45-54

Singh SK, Srivastava PK, Gupta M, Mukherjee S (2012) Modeling mineral phase change chemistry of groundwater in a rural-urban fringe. Water Sci Technol 66:1502-1510

Singh SK, Srivastava PK, Pandey AC, Gautam SK (2013) Integrated assessment of groundwater influenced by a confluence river system: concurrence with remote sensing and geochemical modelling. Water Resour Manag 27(12):4291-4313. https://doi. org/10.1007/s11269-013-0408-y

Singh SK, Srivastava PK, Singh D, Han D, Gautam SK, Pandey AC (2015) Modeling groundwater quality over a humid subtropical region using numerical indices, earth observation datasets, and X-ray diffraction technique: a case study of Allahabad district, India. Environ Geochem Health 37(1):157-180. https://doi. org/10.1007/s10653-014-9638-z

Soltan ME (1998) Characterisation, classification, and evaluation of some ground water samples in upper Egypt. Chemosphere 37(4):735-745

Soltan ME (1999) Evaluation of ground water quality in Dakhla Oasis (Egyptian Western Desert). Environ Monit Assess 57:157-168

Srivastava PK, Singh SK, Gupta M, Thakur JK, Mukherjee S (2013) Modeling impact of land use change trajectories on groundwater quality using remote sensing and GIS. Environ Eng Manag J 12(12):2343-2355

Szabolcs I, Darab C (1964) The influence of irrigation water of high sodium carbonate content of soils. In: Proceedings of 8th international congress of ISSS, Transaction II, pp 803-881

Todd DK (1980) Groundwater hydrology, 2nd edn. Wiley, New York, p 535

United States Environmental Protection Agency (U.S. EPA) (1986) Quality criteria for water 1986, 1 May 1986, EPA 440/5-86-001

Vasanthavigar M, Srinivasamoorthy K, Vijayaragavan K, Rajiv Ganthi R, Chidambaram S, Sarama VS, Anandhan P, Manivannan R, Vasudevan S (2010) Application of water quality index for groundwater quality assessment: Thirumanimuttar Sub-Basin, Tamilnadu, India. Environ Monit Assess 171(1-4):595-609

Wilcox LV (1948) The quality of water for irrigation use. US Department of Agricultural Technical Bulletin 1962, Washington

Publisher's Note Springer Nature remains neutral with regard to jurisdictional claims in published maps and institutional affiliations. 